IP Periodica Polytechnica Electrical Engineering and Computer Science

61(3), pp. 279-295, 2017

https://doi.org/10.3311/PPee.8607

Creative Commons Attribution (i)

RESEARCH ARTICLE

\section{Regularization of the Spatial Inverse Positioning Problem of Revolute Joint Manipulators}

\author{
Dániel András Drexler ${ }^{1 *}$
}

Received 24 September 2015; accepted after revision 11 September 2016

\begin{abstract}
Inverse kinematics is a central problem in robotics, and its solution is burdened with kinematic singularities, i.e. the task Jacobian of the problem is singular. A subproblem of the general inverse kinematics problem, the inverse positioning problem is considered for spatial manipulators consisting of revolute joints, and a regularization method is proposed that results in a regular task Jacobian in singular configurations as well, provided that the manipulator's geometry makes movement in singular directions possible. The conditions of regularizability are investigated, and bounds on the singular values of the regularized task Jacobian are given that can be used to create stable closed-loop inverse kinematics algorithms. The proposed method is demonstrated on the inverse positioning problem of an elbow manipulator and compared to the Damped Least Squares and the Levenberg-Marquardt methods, and it is shown that only the proposed method can leave the singular configuration in the singular direction.
\end{abstract}

\section{Keywords}

singularity, inverse kinematics, Lie algebra, regularization
${ }^{1}$ EKIK Physiological Controls Group, Óbuda University, Budapest, Hungary

${ }^{*}$ Corresponding author, e-mail: drexler.daniel@nik.uni-obuda.hu

\section{Introduction}

Inverse kinematics in general means finding the joint variables of the robot required to reach the desired position and (or) orientation of a specific point or frame of the robot, for example the frame attached to the end effector [1-3]. In most industrial applications the analytical inverse is used, but the analytical solution to the inverse kinematics problem only exists for manipulators with special architecture.

For general architectures, e.g. redundant manipulators [4], the differential inverse kinematics algorithm should be used that calculates the required joint velocities for the desired end effector velocities using the inverse of the analytical Jacobian of the manipulator's forward kinematics map. However, the inverse kinematics problem is burdened with singular configurations, in which the Jacobian matrix becomes singular. Singularities get great attention in the literature, analysis of singularities of robot manipulators is done e.g. in the works of Donelan $[5,6]$ and Kieffer [7].

Generally singularities are either avoided [8,9], or they are handled using numerical methods. Avoiding singularities results in robot motion that neglects some part of the workspace, and the utilization of singular configurations (e.g. gaining mechanical advantage in singularities) becomes impossible if singularities are avoided.

A popular way to handle singularities is to use the MoorePenrose pseudoinverse $J^{\top}\left(J J^{\top}\right)^{-1}$ of the Jacobian $J$ and modify the term $J J^{\top}$ by adding $\lambda I$ with $I$ being the identity matrix of appropriate size to make it invertible. This method is called the Damped Least Squares (DLS) method and used widely in the literature [10-14]. The main disadvantage of this method is that it regularizes the $\left(J J^{\top}\right)$ term instead of the Jacobian itself so the inverse will not be full rank, as it will be shown in Section 3.

Another significant numerical method that works by slightly modifying the calculation of the inverse of the Jacobian is the Levenberg-Marquardt (LM) method [15]. A modified LM method was proposed by Sugihara [16], and it was shown that it has better performance than the numerical inverse kinematics schemes with similar complexity. 
However, the LM method also regularizes the $J J^{\top}$ term in the pseudoinverse, so the inverse mapping is not guaranteed to be full rank, similarly to the case when the DLS method is applied.

In this paper a method is discussed that handles singularities in the inverse positioning problem of serial revolute joint manipulators by regularizing the task Jacobian. The regularization of the inverse positioning problem of planar manipulators has already been discussed [17], while the spatial case was discussed in $[18,19]$. The regularization method regularizes directly the task Jacobian and not its pseudoinverse, so the inverse mapping is guaranteed to be full rank, and motion in singular direction can be generated by the inverse kinematics schemes using the regularized Jacobian. The conditions of regularizability are given, and the numerical properties of the regularized Jacobian are investigated.

In order to regularize the Jacobian, one needs a regularization vector. A theorem is given that helps finding the regularization vector, and a suitable regularization vector is given for elbow manipulators. The regularization of the inverse positioning problem of an elbow manipulator is demonstrated symbolically and numerically.

The mathematical background used in the work is briefly summarized in Section 2. The robot kinematics is described using the Lie algebra of rigid body motion. The positioning problem is discussed in Section 3, where we introduce the end effector Jacobian that is the analytical Jacobian of the forward kinematics map formulated using the Lie algebra of rigid body motion, and we discuss the singularities and the concept of regularization.

The regularization method is generalized to the spatial case for manipulators with three revolute joints in Section 4. Note that manipulators with three revolute joints will be called $3 R$ manipulators, while manipulators with $n$ revolutejoints will be called $n R$ manipulators throughout the paper. We give bounds on the singular values of the regularized Jacobian. We use these bounds to give a stable closed-loop inverse kinematics (CLIK) algorithm [20] in Section 5.

We carry out simulations on an elbow manipulator performing tasks in which the manipulator has to move in and out of typical singular configurations. We use the CLIK algorithm [20] with the proposed regularization method, the DLS method and the modified LM method [16]. We show that only the proposed method can move out of the initial singular configuration if the manipulator has to move in a singular direction. For comparison purposes, the simulation is repeated with the initial configuration being regular, so that the DLS and LM methods give evaluable results. In this case, the proposed method gives similar results like the DLS and LM methods.

\section{Kinematics}

In this section we give a brief overview of the mathematical background of the work that can be found in $[1,2]$. We define the Lie group of rigid body motions and its Lie algebra that consists of the generators of the motion. We connect the Lie group and Lie algebra to the robotics problem by describing the forward kinematics and forward velocity kinematics of the serial robots using these tools.

\subsection{Lie group and Lie algebra of rigid body motions}

In robotic applications we are interested in rigid body transformations consisting of rotations and translations. Rotations are often represented as matrices describing the transformation between orthonormal right-handed coordinate frames, thus matrices representing rotations are from the Special Orthogonal matrix group $S O(3)=\left\{R \in \mathbb{R}^{3 \times 3}: R R^{\top}=I\right.$, $\left.\operatorname{det} R=1\right\}$ endowed with the matrix product as group operation. Translations are usually represented by three dimensional vectors. Thus a rigid body transformation can be described by a pair $(R, p)$ with $R \in S O(3)$ describing the rotation and $p \in \mathbb{R}^{3}$ describing the translation. In order to represent rigid body transformations as linear transformations, the homogeneous coordinates are used, i.e. the pair $(R, p)$ is represented as the matrix

$$
g=\left(\begin{array}{ll}
R & p \\
0 & 1
\end{array}\right) .
$$

We will also use the notation $g=H(R, p)$ emphasizing that the homogeneous matrix represents the rotation $R$ and translation $p$. The matrix $H(I, p)$ with $I$ being the $3 \times 3$ identity matrix thus represents a pure translation, while $H(I, 0)$ represents a pure rotation. Matrices $\mathrm{g}=H(R, p)$ form the Special Euclidean group $S E(3)=\left\{H(R, p) \in \mathbb{R}^{4 \times 4}: R \in S O(3), p \in \mathbb{R}^{3}\right\}$, endowed with the matrix product as group operation. The groups $S O(3)$ and $S E(3)$ are both Lie groups, thus they are differentiable as a manifold, so we can talk about their infinitesimal generators.

Rotations can be written in the form of a matrix exponential $R=\exp (\hat{\omega} t)$, with $\hat{\omega}$ being a $3 \times 3$ skew-symmetric matrix, and $t$ being a real number. In this sense, the skew-symmetric matrices generate the Special Orthogonal group, and form the special orthogonal Lie-algebra $\operatorname{so}(3)=\left\{\hat{\omega} \in \mathbb{R}^{3 \times 3}, \hat{\omega}=-\hat{\omega}^{\top}\right\}$ with the Lie-bracket being the standard matrix commutator $\left[\hat{\omega}_{1}, \hat{\omega}_{2}\right]=\hat{\omega}_{1} \hat{\omega}_{2}-\hat{\omega}_{2} \hat{\omega}_{1}$. There is an isomorphism [21] (invertible linear transformation respecting the Lie-bracket) between the Lie-algebra of $s o(3)$ and the Lie-algebra of $\mathbb{R}^{3}$ with the cross product as the Lie-bracket. This isomorphism is given by

$$
\hat{\omega}=\left(\begin{array}{ccc}
0 & -\omega_{z} & \omega_{y} \\
\omega_{z} & 0 & -\omega_{x} \\
-\omega_{y} & \omega_{x} & 0
\end{array}\right) \leftrightarrow \omega=\left(\begin{array}{c}
\omega_{x} \\
\omega_{y} \\
\omega_{z}
\end{array}\right)
$$


Following the convention of Murray et al. [1], we will use the notation $\omega$ for vectors in $\mathbb{R}^{3}$, and $\hat{\omega}$ for skew-symmetric matrices resulting from the vector $\omega$ using the isomorphism (2). Note that if $\hat{\omega}$ is created from a unit vector $\omega$, then $R=\exp (\hat{\omega} t)$ is the matrix describing the rotation around the axis $\omega$ with the amount of $t$ radians.

Rigid body transformations can also be written as a matrix exponential $g=\exp (\hat{\xi} t)$, with matrices $\hat{\xi}$ in the form

$$
\hat{\xi}=\left(\begin{array}{ll}
\hat{\omega} & v \\
0 & 0
\end{array}\right)
$$

where $\hat{\omega} \in \operatorname{so}(3), v \in \mathbb{R}^{3}$. Matrices like (3) form the Special Euclidean Lie-algebra $s e(3)=\left\{(\hat{\omega}, v): \hat{\omega} \in \operatorname{so}(3), v \in \mathbb{R}^{3}\right\}$ with the Lie-bracket being the standard matrix commutator. Similar to the case of $s o(3)$, there is an isomorphism between $s e(3)$ and $\mathbb{R}^{6}$, where $\mathbb{R}^{6}$ is endowed with the Lie-product $\times_{6}$ :

$$
\left(\begin{array}{l}
v_{1} \\
\omega_{1}
\end{array}\right) \times\left(\begin{array}{c}
v_{2} \\
\omega_{2}
\end{array}\right)=\left(\begin{array}{c}
\omega_{1} \times v_{2}-\omega_{2} \times v_{1} \\
\omega_{1} \times \omega_{2}
\end{array}\right),
$$

with $v_{1}, v_{2}, \omega_{1}, \omega_{2} \in \mathbb{R}^{3}$. The isomorphism is given by

$$
\hat{\xi}=\left(\begin{array}{cc}
\hat{\omega} & v \\
0 & 0
\end{array}\right) \leftrightarrow \xi=\left(\begin{array}{c}
v \\
\omega
\end{array}\right) .
$$

Similar to the convention in Murray et al. [1], we will use the notation $\xi$ for the vector $\left(v^{\top}, \omega^{\top}\right)^{\top}$ and $\hat{\xi}$ for the matrix created from $\xi$ defined by the isomorphism (5). We will call the vector $v$ the linear velocity generator, the vector $\omega$ the angular velocity generator, while $\xi$ the generator of the rigid body motion. In screw theory the generators $\xi$ are called twists [1], in line geometry, they are called the Plücker coordinates of a line [2], with $\omega$ being the direction vector of the line, and $v$ being the moment of the line. In the remaining of the paper, we do not consider general screw motions, only work with translational and rotational generators, i.e. either $\omega=0$, or if $\omega \neq 0$, then $\omega \perp v$.

Changing the coordinates of a generator $\xi$ can be carried out using the Adjoint transformation. If the generators are written as matrices, then the motion generator $\hat{\xi}$ after the coordinate transformation $g=H(R, p)$ is $A d_{g} \hat{\xi}=g \hat{\xi} g^{-1}$. If the generator is written as a vector, then the motion generator $\xi$ after the coordinate transformation $g=H(R, p)$ is

$$
A d_{H(R, p)} \xi=\left(\begin{array}{cc}
R & \hat{p} R \\
0 & R
\end{array}\right) \xi .
$$

The Adjoint transformation is a Lie-algebra automorphism [21], i.e. it is an invertible linear transformation between identical vector spaces that respects the Lie-bracket. It follows that the matrix in (6) is always full rank for any transformation $g=H(R, p) \in S E(3)$.

\subsection{Serial manipulator kinematics}

In robotics, the generators can be used to describe the motion of the joints of a manipulator. Choose a reference (fixed) coordinate frame, called the spatial frame. We will write every quantity (if not stated otherwise) in this spatial frame. Choose a joint configuration of the manipulator in which the joint variables will be zero. We call this configuration the home configuration following Murray et al. [1]. Note that this configuration is called the reference configuration in Selig's book [2]. Assign a motion generator to each of the joints using the following rules:

- If the joint is a rotational joint, its direction vector is the unit vector $\omega$, and $q$ is an arbitrary point on the joint axis, then its motion generator is $\xi=\left((-\omega \times q)^{\top}, \omega^{\top}\right)^{\top}$.

- If the joint is a prismatic joint, its direction vector being the unit vector $v$, then its motion generator is $\xi=\left(v^{\top}, 0^{\top}\right)^{\top}$.

Let $g(0)$ be the homogeneous transformation matrix defining the transformation between the spatial frame and the frame attached to the end effector of the manipulator in the home configuration. Let the manipulator have $n$ joints, let $\xi_{i}$ be the motion generator and $\theta_{i}$ be the joint variable of the $i$ th joint for $i=1,2, \ldots, n$, and let $\theta=\left(\theta_{1}, \theta_{2}, \ldots, \theta_{n}\right)^{\top}$. Then the transformation between the spatial frame and the end effector frame is defined by the product of exponentials formula

$$
g(\theta)=\exp \left(\hat{\xi}_{1} \theta_{1}\right) \exp \left(\hat{\xi}_{2} \theta_{2}\right) \cdot \ldots \cdot \exp \left(\hat{\xi}_{n} \theta_{n}\right) g(0) .
$$

This formula is the solution to the forward kinematics problem.

The motion generators of the manipulator given in this way also define the relationship between the joint velocities and the velocities $\left(v_{0}, \omega_{0}\right)$ in the home configuration:

$$
\left(\begin{array}{c}
v_{0} \\
\omega_{0}
\end{array}\right)=\underbrace{\left(\begin{array}{lll}
\xi_{1} & \xi_{2} & \ldots \\
\xi_{n}
\end{array}\right)}_{J^{s}(0)}\left(\begin{array}{c}
\dot{\theta}_{1} \\
\dot{\theta}_{2} \\
\vdots \\
\dot{\theta}_{n}
\end{array}\right)
$$

the matrix $J^{s}(0)$ being the spatial manipulator Jacobian in the home configuration. The columns of the spatial manipulator Jacobian in a general joint configuration $\theta$ can be calculated using the Adjoint transformation

$$
\begin{aligned}
J^{s}(\theta) & =\left(\begin{array}{llll}
\xi_{1}^{\prime} & \xi_{2}^{\prime} & \ldots & \xi_{n}^{\prime}
\end{array}\right) \\
\xi_{i}^{\prime} & =A d_{\exp \left(\hat{\xi}_{1} \theta_{1}\right) \exp \left(\hat{\xi}_{2} \theta_{2}\right) \ldots \exp \left(\hat{\xi}_{i-1} \theta_{i-1}\right)} \xi_{i} .
\end{aligned}
$$

The angular velocity $\omega_{0}$ defined in (8) is the angular velocity of the frame attached to the end effector of the manipulator, however the linear velocity $v_{0}$ given in (8) is not the linear velocity of the end effector, but the linear velocity of the (not necessarily real) point of the rigid body moving through the origin of the spatial frame, also being emphasized in Murray et al. [1]. Thus the range space of the spatial manipulator Jacobian 
is the linear velocity of the point at the origin of the spatial frame and the angular velocity of the end effector frame both defined in the fixed spatial frame.

If we transform the motion generators into the frame attached to the end effector, we get the body manipulator Jacobian that can be calculated using the Adjoint transformation as

$$
J^{b}(\theta)=A d_{g(\theta)}^{-1} J^{s}(\theta)
$$

with $g(\theta)$ being the solution of the forward kinematics problem in the joint configuration $\theta$ given by (7). The range space of $J^{b}$ is the linear velocity of the end effector frame origin and the angular velocity of the end effector frame, both defined in the end effector frame.

\section{The positioning subproblem \\ 3.1 The task Jacobian}

A typical problem in robotics is to find the joint variables necessary to reach the desired end effector position. The problem can be solved locally in terms of velocities: given the desired end effector velocity, one needs to find the required joint velocities. The problem can be solved using the linear velocity generators assigned to the joints. The linear velocity generator component of a motion generator $\xi$ will be denoted by $\xi_{V}$, and the matrix of linear velocity generator components of the Jacobian $J^{s}$ will be denoted by $J_{V}^{s}$, that consists of the first three rows of $J^{s}$.

The linear velocity generators of the columns of the spatial manipulator Jacobian describe the linear velocity of the point at the origin of the spatial frame and not the linear velocity of the end effector frame. However, for the inverse positioning task, the linear velocity generators of the end effector frame are needed. This problem can be solved in two ways: either use the body Jacobian, however in this case every velocity has to be given in the end effector frame, or use the action point transformation [17] to redefine the action point of the velocity generator.

Let $\xi$ be a motion generator associated to a robot joint (i.e. some column of the spatial Jacobian), and $p$ be the position vector of a point on the rigid body moved by the robot joint. Then

$$
\xi^{\prime}=\operatorname{Ad}_{H(I,-p)} \xi
$$

is the motion generator whose linear velocity generator component $\left(\xi_{V}^{\prime}\right)$ describes the linear velocity of the point $p$ in the spatial frame. The transformation (12) is called the action point transformation, that can be written regarding only the linear velocity generator of the result as [17]

$$
v^{\prime}=v+\omega \times p
$$

provided that $\xi=\left(v^{\top}, \omega^{\top}\right)^{\top}$ and $\xi^{\prime}=\left(v^{\prime \top}, \omega^{\top}\right)^{\top}$ (note that the action point transformation leaves the angular velocity generator unaffected).
Using the action point transformation, we define the end effector Jacobian $J^{e}$, whose columns are the linear and angular velocity generators of the end effector frame given in the spatial frame. If $p(\theta)$ is the position vector and $R(\theta)$ is the matrix describing the orientation of the end effector frame in the joint configuration $\theta$ given in the spatial frame, then the end effector Jacobian can be calculated as

$$
J^{e}(\theta)=\operatorname{Ad}_{H(I,-p(\theta))} J^{s}(\theta)
$$

or

$$
J^{e}(\theta)=\operatorname{Ad}_{H(R(\theta), 0)} J^{b}(\theta)
$$

Theorem 1. The ranks of the spatial manipulator Jacobian $J^{s}(\theta)$, body manipulator Jacobian $J^{b}(\theta)$ and the end effector Jacobian $J^{e}(\theta)$ are identical in every joint configuration $\theta \in \mathbb{R}^{n}$.

Proof. The matrices are connected by Adjoint transformations as defined by the formulas (11), (14) and (15). Since the Adjoint transformations are Lie-algebra automorhpism, they are invertible, thus leave the ranks of the matrices unchanged.

The task Jacobian of the inverse positioning problem is thus the Jacobian $J_{V}^{e}$ that consists of the linear velocity generators of the end effector Jacobian. For the sake of simplicity, we will also use the notation $J:=J_{V}^{e}$ later in the article.

\subsection{Singular configurations}

In a certain configuration $\theta \in \mathbb{R}^{n}$, the local solution of the inverse positioning task lies in finding the $\dot{\theta}$ joint velocities required for the desired end effector motion $v_{e}$, i.e. by solving the linear system of equations

$$
v_{e}=J(\theta) \dot{\theta}
$$

which is the first-order approximation of the motion of the end effector, that is the first-order approximation of the Taylor-series of the forward kinematics map at the joint configuration $\theta$. If the equations in (16) become linearly dependent, i.e. $J$ drops rank, then we call that situation a singular configuration. In this configuration, first-order motion in some directions (called the singular directions) is not possible. The robot can also withstand theoretically infinite forces or torques acting from the singular directions. However, higher-order motion may be possible in that direction. There are four cases:

1. Motion in singular direction results in continuous joint path but infinite joint velocity, see e.g. Fig. 1.

2. Motion in singular direction results in discontinuous joint path, see e.g. Fig. 2.

3. Motion in singular direction is not possible; in the current configuration, motion is constrained to a lower dimensional manifold, see e.g. Fig. 3.

4. Motion in singular direction is not possible in any 
configuration (trivial singularity), e.g. translation parallel to the joint axis of a planar manipulator. This singularity can be excluded by the appropriate formalization of the task space.

In the first two cases, motion in singular direction is possible, however the solution of (16) does not contain motion component in that direction, so motion in singular direction can not be generated as the solution of the system of equations.

A typical solution to overcome singularities is to use the Moore-Penrose pseudoinverse of the task Jacobian to solve the system of equations (16), i.e. calculate the joint velocities as

$$
\dot{\theta}=J^{\top}\left(J J^{\top}\right)^{-1} v_{e}
$$

and regularize the term $J J^{\top}$ by adding the distortion term $\lambda I$. The resulting pseudoinverse is $J^{\top}\left(J J^{\top}+\lambda I\right)^{-1}$, and this is called the Damped Least Squares (DLS) method. However, application of the DLS method does not necessarily result in motion in singular direction, since the mapping $J^{\top}\left(J J^{\top}+\lambda I\right)$ is still rank deficient. This can be shown by using the theorem on matrix product ranks: the rank of a matrix product can not be greater than the rank of the terms in the product, so the rank of the inverse mapping can not be greater that the rank of $J$. As a consequence of this rank deficiency, if the desired end effector velocity is a vector from the singular direction, then the DLS method will generate no end effector motion.

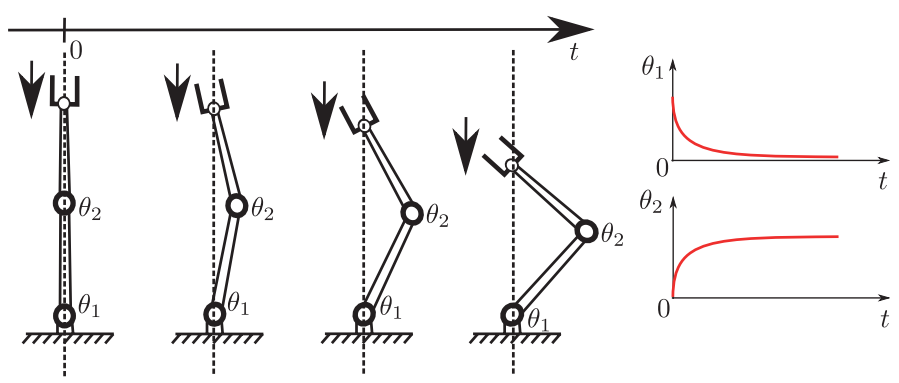

Fig. 1 Motion of a planar arm in the direction denoted by the arrow. The direction is singular in the initial configuration of the robot (at $t=0$ ), however motion is possible with continuous joint paths, but the joint velocities are infinite at the singularity (the tangents of the joint paths are vertical at $t=0$ ).

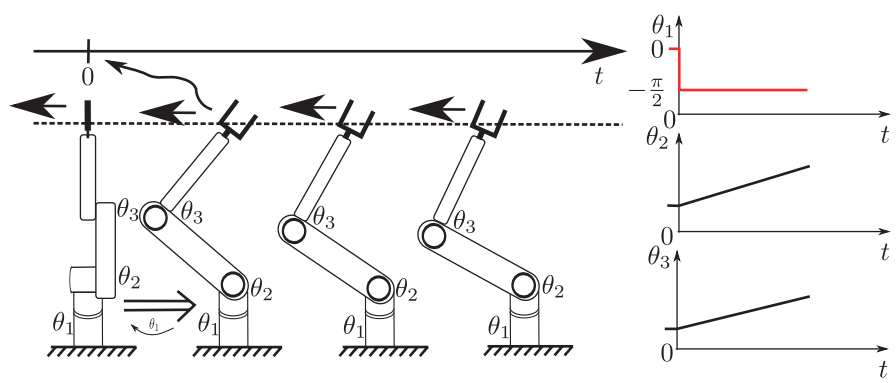

Fig. 2 Motion of an elbow manipulator in the direction denoted by the arrow. The direction is singular in the initial configuration of the robot (at $t=0$ ), and the arm has to take a $-\pi / 2$ turn in its first joint to make motion in the desired direction be possible, so the joint path of $\theta_{1}$ is discontinuous at the singularity (at $t=0$ ).

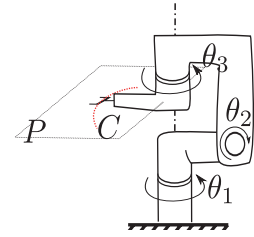

Fig. 3 A fictive manipulator in a singular configuration; the end effector can not reach the points in plane $P$ except for the points on arc $C$.

\subsection{Regularization of the inverse positioning problem}

However, if the matrix $J$ was regularized instead of the term $J J^{\top}$, then the inverse mapping would be full rank. A regularization method was proposed in [17] for the inverse positioning problem of planar manipulators that regularizes the task Jacobian. The concept of the regularization lies in perturbing the action point of the velocity generators by virtually translating the end effector in the direction $r$ at the distance $\gamma$. The infinitesimal motion of the new point will be approximately the same as the infinitesimal motion of the original end effector point, however its linear velocity generators will be linearly independent.

Fig. 4 shows the concept of the regularization for the planar case. Fig. 4(a), shows the planar manipulator, $q_{1}$ and $q_{2}$ being points on the two joint axes of the manipulator, the joint axes being perpendicular to the plane of the robot, $p(0)$ being the position vector of the end effector in the home configuration, and $l_{1}$ and $l_{2}$ being the lengths of the segments of the robot.

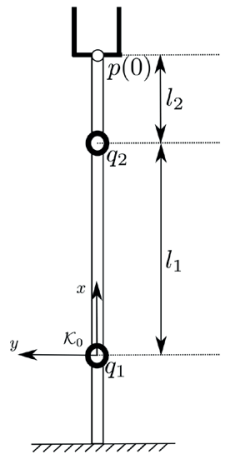

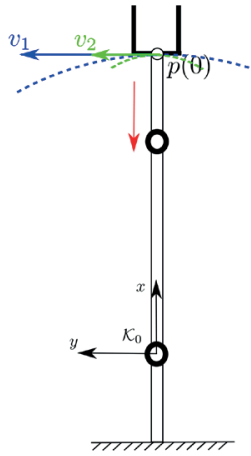

$b$

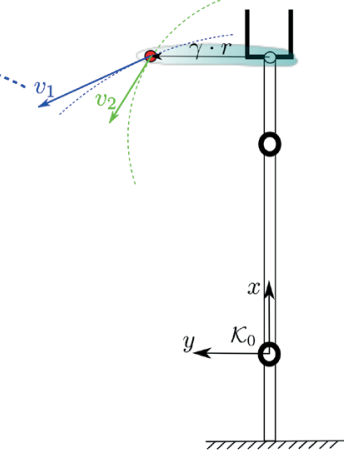

$c$,
Fig. 4 Illustration of the regularization of the inverse positioning problem of a planar manipulator. (a) The geometrical parameters of the planar manipulator. (b) The linear velocity generators in the singular configuration, the generators are parallel. (c) Translating the action point in the direction $r$ at distance $\gamma$, the new linear velocity generators are linearly independent.

The linear velocity generator assigned to a joint and the point $p(0)$ can be geometrically visualized as a vector with its direction vector being the tangent of the circle on which the joint moves the point $p(0)$ at the point $p(0)$, and its magnitude being the distance of the point $p(0)$ and the joint axis. The linear velocity generators of the end effector assigned to the 
joints are the $v_{1}$ and $v_{2}$ vectors in Fig. 4(b). In this example, the points $q_{1}, q_{2}$ and $p(0)$ are collinear, thus the linear velocity generators $v_{1}$ and $v_{2}$ are parallel. The singular direction is perpendicular to the linear velocity generators, represented by the vector pointing in the $-x$ direction in Fig. 4(b).

However, if the end effector point is replaced in the direction $r$ at distance $\gamma$ as in Fig. 4(c), then the linear velocity generators of the new point (the red point in Fig. 4(c)) will be linearly independent. If the new point is considered to be rigidly attached to the end effector, then the infinitesimal motion of the perturbed point will be approximately the same as the infinitesimal motion of the end effector. This method is called the regularization method [17], and the Jacobian containing the linear velocity generators of the new point is the regularized Jacobian $J^{\text {reg }}$. This Jacobian can be used instead of $J$ to solve (16).

In the spatial case, the regularization can be defined similarly: the action point of the linear velocity generators of the end effector Jacobian is translated in the direction $r$ at the distance $\gamma$ to get the regularized Jacobian

$$
J^{\text {reg }}=\left(\operatorname{Ad}_{H(I,-\gamma r)} J^{e}\right)_{V}
$$

Note that with the $V$ subscript we denote choosing the linear velocity generator components, i.e. the first three rows of the matrix (or a generator $\xi$ ). We will also use the $\Omega$ subscript to denote choosing the angular velocity generators, i.e. the last three rows of the matrix (or a generator $\xi$ ).

The regularizability in the spatial case is further analyzed in the next Section.

4 Regularizability of the inverse positioning problem

Definition 1. The inverse positioning problem of a spatial manipulator is regularizable in a joint configuration $\theta \in \mathbb{R}^{3}$ if there exists $r \in \mathbb{R}^{3}$ and $\gamma \in \mathbb{R}^{3}$ such that

$$
J^{\mathrm{reg}}(\theta)=\left(\operatorname{Ad}_{H(I,-\gamma r)} J^{e}(\theta)\right)_{V}
$$

is full rank.

Since the subscript $V$ in (19) denotes choosing the linear velocity generators, we only have to consider the resulting linear velocity generators while calculating $J^{\text {reg }}$. The action point transformation considering only linear velocity generator of the result acts as in (13), thus the regularized Jacobian can be written as

$$
\begin{gathered}
J^{\mathrm{reg}}=\left(\begin{array}{llll}
v_{1}{ }^{\text {reg }} & v_{2}{ }^{\text {reg }} & \ldots & v_{n}{ }^{\text {reg }}
\end{array}\right) \\
v_{i}^{\text {reg }}=v_{i}^{e}+\gamma \omega_{i}^{e} \times r, \quad i=1,2, \ldots, n .
\end{gathered}
$$

Utilizing the notations

and

$$
J_{V}^{e}=\left(\begin{array}{llll}
v_{1}^{e} & v_{2}^{e} & \ldots & v_{n}^{e}
\end{array}\right)
$$

$$
J_{\Omega}^{e}=\left(\begin{array}{llll}
\omega_{1}^{e} & \omega_{2}^{e} & \ldots & \omega_{n}^{e}
\end{array}\right)
$$

and introducing the notation $J_{\Omega}^{e} \times r$ to denote columnwise vector product, i.e.

$$
J_{\Omega}^{e} \times r=\left(\begin{array}{llll}
\omega_{1}^{e} \times r & \omega_{2}^{e} \times r & \ldots & \omega_{n}^{e} \times r
\end{array}\right),
$$

we can write the regularized Jacobian as

$$
J^{\mathrm{reg}}=J_{V}^{e}+\gamma J_{\Omega}^{e} \times r .
$$

Definition 2. A vector $r \in \mathbb{R}^{3}$ is called a regularization vector if it regularizes the inverse positioning problem, i.e. the matrix in (19) is full rank for some $\gamma \in \mathbb{R}^{3}$.

Determining the regularization vector for a given problem is crucial if we want to carry out regularization according to (19). Next, we prove, that the regularization vector needs to have a component from the image space of the task Jacobian.

Theorem 2. Any regularization vector has a component that is in the image space of the task Jacobian.

Proof. Since the regularized Jacobian is

$$
J^{\text {reg }}=J_{V}^{e}+\gamma J_{\Omega}^{e} \times r
$$

its range space has the property

$$
\operatorname{Ran} J^{\text {reg }} \subseteq \operatorname{Ran} J_{V}^{e} \cup \operatorname{Ran}\left(J_{\Omega}^{e} \times r\right)
$$

where $\cup$ is the subspace union. Suppose indirectly, that $r \notin \operatorname{Ran} J_{V}^{e}$. Note that this implies $r \neq 0$, since the zero vector is element of every subspace. Since $J_{\Omega}^{e} \times r$ is orthogonal to $r$, $r \notin \operatorname{Ran}\left(J_{\Omega}^{e} \times r\right)$, and $r \notin \operatorname{Ran} J^{\text {reg }}$ follows because $r$ is not in any subspace on the right-hand side of (27). This implies that $J^{\text {reg }}$ is not full rank, that contradicts with the statement that $r$ is a regularization vector.

In the following theorem we give a necessary condition for regularizability.

Theorem 3. If the task Jacobian of a $3 \mathrm{R}$ manipulator is regularizable, then the end effector Jacobian is full rank.

Proof. Since the regularized Jacobian by definition is

$$
J^{\text {reg }}=\left(\operatorname{Ad}_{H(I,-\gamma r)} J^{e}\right)_{V},
$$

it can be written as

$$
J^{\text {reg }}=\pi_{V} \operatorname{Ad}_{H(I,-\gamma r)} J^{e},
$$

where $\pi_{V}$ is the projector matrix

$$
\pi_{V}=\left(\begin{array}{llllll}
1 & 0 & 0 & 0 & 0 & 0 \\
0 & 1 & 0 & 0 & 0 & 0 \\
0 & 0 & 1 & 0 & 0 & 0
\end{array}\right) .
$$

Since the rank of $J^{\text {reg }}$ can not be greater than the minimum of the rank of the matrices in the right-hand side of (29), and $\operatorname{rank} \pi_{V}=3$, while $\operatorname{rankAd} A_{H(I,-\gamma r)}=6$ (since Ad 
is an automorphism of six-dimensional vector spaces), and $\operatorname{rank} J^{e} \leq 3$, the rank of $J^{\text {reg }}$ is

$$
\begin{aligned}
\operatorname{rank} J^{\mathrm{reg}} & \leq \min (\underbrace{\operatorname{rank} \pi_{V}}_{=3}, \underbrace{\operatorname{Ad}_{H(I,-\gamma r)}}_{=6}, \operatorname{rank} J^{e}) \\
& =\operatorname{rank} J^{e} .
\end{aligned}
$$

So $\operatorname{rank} J^{\text {reg }}=3$ implies that $\operatorname{rank} J^{e}=3$.

There are three classes of $3 \mathrm{R}$ manipulators whose spatial (i.e. three-dimensional) inverse positioning problem is never regularizable. The first class consists of the planar manipulators that can only move in a plane, the second class consists of the manipulators with all the three joint axes intersecting in a point and the third (not necessarily disjoint from the previous) class consists of manipulators that have their end effector frame origin on the last joint axis. The positioning subproblem of these manipulators is always two-dimensional [22], thus we exclude these manipulators from further analysis. Note that the manipulators with intersecting joint axes or with their end effector frame origin being on the last joint axis are usually used to solve the inverse orientation problem [22]. The task Jacobian of the $3 \mathrm{R}$ manipulators that do not belong to the excluded classes may be regularizable with the appropriate choice of the regularization vector.

Theorem 4. The position $p$ of the end effector frame origin lies on the third joint axis $\omega_{3}$ if and only if $v_{3}^{p}=0$.

Proof. The linear velocity generator is $v_{3}^{p}=\omega_{3} \times\left(p-q_{3}\right)$ (by the definition and the application of the action point transformation [17]), where $q_{3}$ is a point on the joint axis, but since $v_{3}^{p}=0$, the equality $\omega_{3} \times\left(p-q_{3}\right)=0$ holds. This equality means that $\lambda \omega_{3}=\left(p-q_{3}\right)$ for any $\lambda \in \mathbb{R}^{3}$, that can be rearranged to get $p=\lambda \omega_{3}+q_{3}$, which means that $p$ is on the third joint axis. The opposite direction of the proof is done by reversing the proof.

So if the end effector frame origin is not on the last joint axis, then $v_{3}^{e} \neq 0$. Next we investigate the kernels of the Jacobian $J_{V}^{e}$ in the following two Lemmas.

Lemma 1. The joint axes of a $3 R$ manipulator intersect at the same point if and only if there exists a point such that if this point is considered the end effector point $p(\theta)$, then the end effector Jacobian is $J_{V}^{e}=0$.

Proof. If the joint axes of the manipulators intersect at some point $q_{1}(\theta)=q_{2}(\theta)=q_{3}(\theta):=q(\theta)$ in the joint configuration $\theta \in \mathbb{R}^{3}$, while the end effector position is chosen to be $p(\theta):=q(\theta)$, then the linear velocity generators are defined by

$$
\begin{aligned}
& v_{1}^{e}(\theta)=\omega_{1}^{e}(\theta) \times\left(p(\theta)-q_{1}(\theta)\right)=0 \\
& v_{2}^{e}(\theta)=\omega_{2}^{e}(\theta) \times\left(p(\theta)-q_{2}(\theta)\right)=0 \\
& v_{3}^{e}(\theta)=\omega_{3}^{e}(\theta) \times\left(p(\theta)-q_{3}(\theta)\right)=0
\end{aligned}
$$

so the linear velocity generators are all zero, thus $J_{V}^{e}=0$.

Suppose that there exists a point such that $J_{V}^{e}=0$ in the joint configuration $\theta \in \mathbb{R}^{3}$ if that point is chosen to be the end effector point. Let this point be $p(\theta)$. Since $J_{V}^{e}=0$, this implies that (32)-(34) hold. So for every joint $i \in\{1,2,3\}$, either $\omega_{i}^{e}(\theta)$ is parallel to $\left(p(\theta)-q_{i}(\theta)\right)$ or $p(\theta)=q_{i}(\theta)$. If $p(\theta)=q_{i}(\theta)$ that means $p(\theta)$ is on the $i$ th joint axis. If $\omega_{i}^{e}(\theta)$ is parallel to $\left(p(\theta)-q_{i}(\theta)\right)$ that also mean $p(\theta)$ is on the $i$ th joint axis, since $q_{i}(\theta)$ is on the $i$ th joint axis. So $p(\theta)$ is a point on all joint axes, thus the joint axes intersect in the same point that is exactly $p(\theta)$.

The consequence of this Lemma is that the rank of the task Jacobian is always greater than zero if the joint axes of the manipulator do not intersect at the same point, and this property is invariant of the action point transformation.

Next we show, that if the end effector Jacobian is full rank, then there is no nonzero vector that is in the kernel of both $J_{V}^{e}$ and $J_{\Omega}^{e}$.

Lemma 2. If $J^{e}$ is the end effector Jacobian of a 3R manipulator, and $\operatorname{rank} J^{e}=3$, then there exists no nonzero $\tau \in \mathbb{R}^{3}$, such that $\tau \in \operatorname{Ker} J_{V}^{e}$ and $\tau \in \operatorname{Ker} J_{\Omega}^{e}$.

Proof. Suppose indirectly, that there exists a nonzero $\tau \in \mathbb{R}^{3}$, such that both $\tau \in \operatorname{Ker} J_{V}^{e}$ and $\tau \in \operatorname{Ker} J_{\Omega}^{e}$ hold. Since the end effector Jacobian is

$$
J^{e}=\left(\begin{array}{c}
J_{V}^{e} \\
J_{\Omega}^{e}
\end{array}\right)
$$

it follows that $\tau$ is in the kernel of $J^{e}$, since

$$
J^{e} \tau=\left(\begin{array}{c}
J_{V}^{e} \\
J_{\Omega}^{e}
\end{array}\right) \tau=\left(\begin{array}{l}
J_{V}^{e} \tau \\
J_{\Omega}^{e} \tau
\end{array}\right)=\left(\begin{array}{l}
0 \\
0
\end{array}\right)=0,
$$

which implies that $J^{e}$ is not full rank, that is a contradiction.

Next, we define the $Q$ matrix that will be useful in the proof of Theorem 5 as

$$
Q=\frac{1}{2}\left(\left(J_{V}^{e}\right)^{\top} J_{\Omega}^{e}+\left(J_{\Omega}^{e}\right)^{\top} J_{V}^{e}\right)
$$

An invariant bilinear form of the special Euclidean Lie-algebra is the reciprocal product

$$
\xi_{1} \odot \xi_{2}=\omega_{1}^{\top} v_{2}+\omega_{2}^{\top} v_{1}
$$

The reciprocal product is nondegenarate, i.e. there exists no nonzero $\xi^{\prime}$ such that $\xi^{\prime} \odot \xi=0$ for every $\xi \in \mathbb{R}^{6}$. The reciprocal product of two rotational generators is zero if and only if their axes of rotations are parallel or they intersect in a point [2]. 
It can be shown with direct calculation that for $3 R$ manipulators the matrix in (37) can also be written as

$$
Q=\frac{1}{2}\left(\begin{array}{ccc}
0 & \xi_{1}^{e} \odot \xi_{2}^{e} & \xi_{1}^{e} \odot \xi_{3}^{e} \\
\xi_{1}^{e} \odot \xi_{2}^{e} & 0 & \xi_{2}^{e} \odot \xi_{3}^{e} \\
\xi_{1}^{e} \odot \xi_{3}^{e} & \xi_{2}^{e} \odot \xi_{3}^{e} & 0
\end{array}\right)
$$

with $\odot$ denoting the reciprocal product defined by (38). Note that if the $3 \mathrm{R}$ manipulator is a planar manipulator (all joint axes are parallel), or all of its three joint axes intersect in the same point (the manipulator is spherical), then $Q=0$.

However $Q=0$ may also occur if two of the joint axes are parallel, while the third joint axis intersects the other two joint axes, which is the case e.g. if an elbow manipulator is in a corank two singular configuration (in a configuration where the rank of the Jacobian drops by two, see e.g. the second manipulator in Fig. 5, or the manipulator in Fig. 6).

Lemma 3. The $Q$ matrix has the following properties:

1. $\operatorname{det} Q=\left(\xi_{1}^{e} \odot \xi_{2}^{e}\right)\left(\xi_{2}^{e} \odot \xi_{3}^{e}\right)\left(\xi_{1}^{e} \odot \xi_{3}^{e}\right)$.

2. If $Q \neq 0$, then $\operatorname{rank} Q \geq 2$.

Proof.

1) The determinant of the matrix (39) can be written as

$$
\begin{aligned}
\operatorname{det} Q= & -\frac{1}{2} \xi_{1}^{e} \odot \xi_{2}^{e}\left(0-\left(\xi_{1}^{e} \odot \xi_{3}^{e}\right)\left(\xi_{2}^{e} \odot \xi_{3}^{e}\right)\right) \\
& +\frac{1}{2} \xi_{1}^{e} \odot \xi_{3}^{e}\left(\left(\xi_{1}^{e} \odot \xi_{2}^{e}\right)\left(\xi_{2}^{e} \odot \xi_{3}^{e}\right)-0\right) \\
= & \left(\xi_{1}^{e} \odot \xi_{2}^{e}\right)\left(\xi_{2}^{e} \odot \xi_{3}^{e}\right)\left(\xi_{1}^{e} \odot \xi_{3}^{e}\right) .
\end{aligned}
$$

2) If any element of the $Q$ matrix is not zero, then it has two columns with different coordinates that are zero (because of the zero diagonal of the matrix), and different coordinates that are nonzero (because of the nonzero element of the symmetric matrix), so they are linearly independent, which implies that the rank of the matrix is at least two.

Lemma 4. Let $Q \neq 0$ be a $3 \times 3$ symmetric real matrix with zero elements in the diagonal, and $S$ be a proper subspace of $\mathbb{R}^{3}$ with $\operatorname{dim} S \leq 2$, and $\lambda \in \mathbb{R}^{3}$. Then the solution of the quadratic equation $<Q \lambda, \lambda>=0$ restricted to $S$ is one of the following:

1. A line passing through the origin.

2. The origin.

3. Two lines passing through the origin.

Proof. Let $P_{S}$ be the orthogonal projection to the subspace $S$, then $\operatorname{dim} S \leq 2 \operatorname{implies} \operatorname{rank} P_{S} \leq 2$. The variables $\lambda$ restricted to $S$ are $P_{S} \lambda$, so the equation $<Q \lambda, \lambda>=0$ restricted to $S$ can be written as

$$
<Q P_{S} \lambda, P_{S} \lambda>=<P_{S}^{\top} Q P_{S} \lambda, \lambda>=0 .
$$

Introducing $Q_{S}=P_{S}^{\top} Q P_{S}$, the quadratic equation restricted to $S$ becomes

$$
<Q_{S} \lambda, \lambda>=0 .
$$

Since $Q \neq 0$ is symmetric with zero diagonal, its rank is at least two by Lemma 3, while $\operatorname{rank} P_{S} \leq 2$, so by the rank nullity theorem and the property of product rank, $1 \leq \operatorname{rank} Q_{S} \leq 2$. Moreover, $Q_{S}$ is also a symmetric real matrix, so its eigenvalues are real, and it is diagonalizable, i.e. it can be written in the form

$$
Q_{S}=U\left(\begin{array}{ccc}
\kappa_{1} & 0 & 0 \\
0 & \kappa_{2} & 0 \\
0 & 0 & 0
\end{array}\right) U^{\top}
$$

with $U U^{\top}=I$, and $\kappa_{1}, \kappa_{2} \in \mathbb{R}$. Substituting this form into the quadratic equation results in

$$
\lambda^{\top} U^{\top}\left(\begin{array}{ccc}
\kappa_{1} & 0 & 0 \\
0 & \kappa_{2} & 0 \\
0 & 0 & 0
\end{array}\right) U \lambda=0 .
$$

Introducing the rotated variables $\tilde{\lambda}=\left(\tilde{\lambda}_{1}, \tilde{\lambda}_{2}, \tilde{\lambda}_{3},\right)^{\top}$ defined as $\tilde{\lambda}=U \lambda$, the quadratic equation becomes

$$
\left(\begin{array}{lll}
\tilde{\lambda}_{1} & \tilde{\lambda}_{2} & \tilde{\lambda}_{3}
\end{array}\right)\left(\begin{array}{ccc}
\kappa_{1} & 0 & 0 \\
0 & \kappa_{2} & 0 \\
0 & 0 & 0
\end{array}\right)\left(\begin{array}{c}
\tilde{\lambda}_{1} \\
\tilde{\lambda}_{2} \\
\tilde{\lambda}_{3}
\end{array}\right)=0
$$

that reduces to

$$
\kappa_{1} \tilde{\lambda}_{1}^{2}+\kappa_{2} \tilde{\lambda}_{2}^{2}=0
$$

Note that since the vector $\left(0,0, \tilde{\lambda}_{3}\right)^{\top} \notin S$ (because $S$ is at most two-dimensional), the subspace $S$ after the transformation $U$ is spanned by $\left(\tilde{\lambda}_{1}, 0,0\right)^{\top}$ and $\left(0, \tilde{\lambda}_{2}, 0\right)^{\top}$.

Suppose that $\kappa_{2}=0$. In this case $\kappa_{1} \neq 0$, because $\kappa_{1}=0$ would imply $Q_{S}=0$ that is a contradiction. The quadratic equation (46) reduces to $\kappa_{1} \tilde{\lambda}_{1}^{2}=0$, and the solution is $\tilde{\lambda}_{1}=0$, $\tilde{\lambda}_{2} \in \mathbb{R}$ that is a line passing through the origin.

Suppose that $\kappa_{1}=0$. In this case $\kappa_{2} \neq 0$, because $\kappa_{2}=0$ would imply $Q_{S}=0$ that is a contradiction. The quadratic equation (46) reduces to $\kappa_{2} \lambda_{2}^{2}=0$ and the solution is $\tilde{\lambda}_{1} \in \mathbb{R}$, $\tilde{\lambda}_{2}=0$ that is a line passing through the origin.

Suppose that $\kappa_{1} \neq 0$ and $\kappa_{2} \neq 0$, and $\kappa_{1} \kappa_{2}>0$, so they have the same sign. Then the only real solution to the quadratic equation (46) is the point $\tilde{\lambda}_{1}=\tilde{\lambda}_{2}=0$ that is the origin.

Suppose that $\kappa_{1} \neq 0$ and $\kappa_{2} \neq 0$, and $\kappa_{1} \kappa_{2}<0$, so they have different sign. Then the only real solution to the quadratic equation (46) $\tilde{\lambda}_{1}= \pm \sqrt{-\kappa_{2} / \kappa_{1}} \tilde{\lambda}_{2}$ is that is the set of two lines passing through the origin.

The matrix $Q$ can be zero if all the joint axis are parallel, all the joint axis intersect at the same point, or some joints intersect and are parallel, but not all. The latter situations are depicted in Fig. 5. 
For the manipulator in the top of Fig. 5, the geometrical parameters can be chosen as $\omega_{1}=(1,0,0)^{\top}, \omega_{2}=(0,1,0)^{\top}$, $\omega_{3}=(1,0,0)^{\top}, \quad q_{1}=0, \quad q_{2}=0, \quad q_{3}=\left(0, l_{1}, 0\right)^{\top}$ and $p_{0}=$ $\left(0, l_{1}+l_{2}, 0\right)^{\top}$. Direct calculation yields that the task Jacobian is

$$
J_{V}^{e}=\left(\begin{array}{ccc}
0 & 0 & 0 \\
0 & 0 & 0 \\
l_{1}+l_{2} & 0 & l_{2}
\end{array}\right)
$$

whose rank is one. It can be easily shown, that the other two manipulators in Fig. 5 also have $\operatorname{rank} J_{V}^{e}=1$. Note that if the end effector points are relocated appropriately, then the task Jacobians may have rank greater than one even with reciprocal joints.
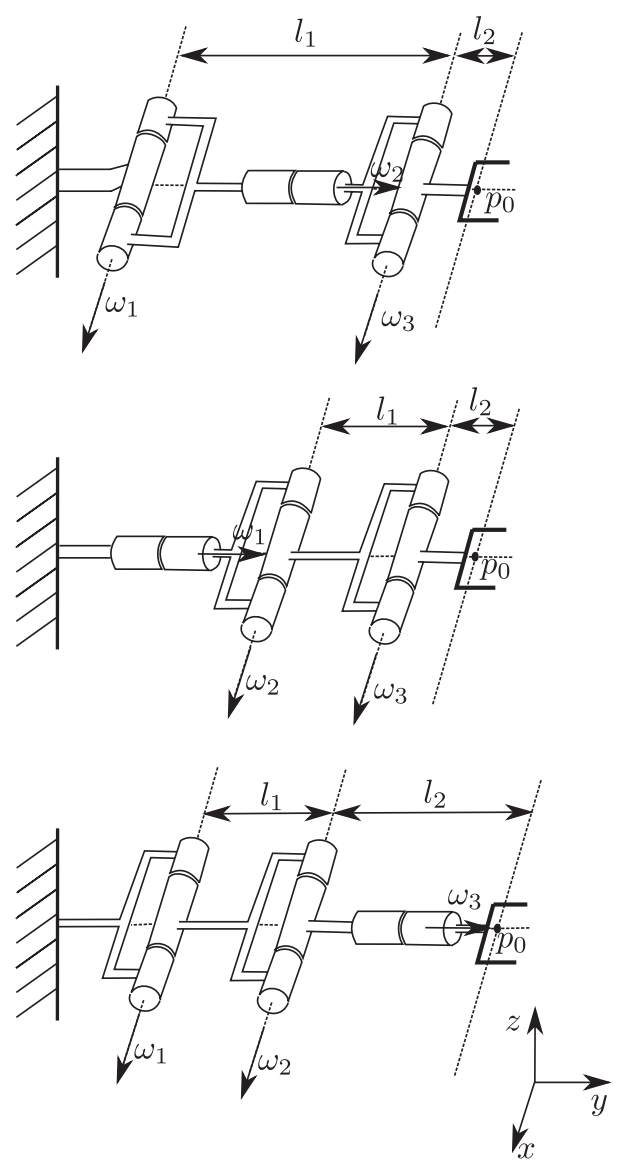

Fig. 5 Manipulator architectures with joint configurations such that all joints are reciprocal, spherical and planar manipulators excluded

Theorem 5. Suppose that a $3 R$ manipulator is not planar, its joint axes do not meet in a point and the end effector frame origin is not on the third joint axis. Then the inverse positioning problem of the manipulator is regularizable in the current joint configuration if and only if its end effector Jacobian $J^{e}$ is full rank in that configuration.

Proof. If $J_{V}^{e}$ is regularizable, then $J^{e}$ is full rank because of Theorem 3, so only the other direction needs to be proved. Suppose that $J^{e}$ is full rank. Then the regularized Jacobian is

$$
J^{\mathrm{reg}}=J_{V}^{e}+\gamma\left(J_{\Omega}^{e} \times r\right)
$$

which is regular if and only if

$$
J^{\text {reg }} \lambda=0
$$

implies $\lambda=0$. It will be proved, that there exists $r \in \mathbb{R}^{3}$ and $\gamma \in \mathbb{R}$, such that (49) holds if and only if $\lambda=0$. Note that $\lambda=0$ is a trivial solution, and throughout the proof, all nonzero $\lambda$ will be analyzed, and it will be shown, that if $\lambda \neq 0$, then for suitably chosen $r \in \mathbb{R}^{3}$ and $\gamma \in \mathbb{R}$, (49) does not hold.

Substituting (48) into the condition (49), it can be rephrased as $J^{\text {reg }}$ is regular if and only if

$$
J_{V}^{e} \lambda+\gamma\left(J_{\Omega}^{e} \lambda\right) \times r=0
$$

implies $\lambda=0$. This condition can be further manipulated to get

$$
J_{V}^{e} \lambda=-\gamma\left(J_{\Omega}^{e} \lambda\right) \times r .
$$

Because of the properties of the vector product, this equation holds if and only if

$$
\begin{gathered}
\left\langle J_{V}^{e} \lambda, r\right\rangle=0 \\
\left\langle J_{V}^{e} \lambda, J_{\Omega}^{e} \lambda\right\rangle=0
\end{gathered}
$$

with $\langle\cdot, \cdot\rangle$ denoting the scalar product and for all $\tilde{\lambda}$ that is the solution of (52) and (53)

$$
\gamma=-\operatorname{sign}\left(\left\langle J_{V}^{e} \tilde{\lambda},\left(J_{\Omega}^{e} \tilde{\lambda}\right) \times r\right\rangle\right) \frac{\left\|J_{V}^{e} \tilde{\lambda}\right\|}{\left\|\left(J_{\Omega}^{e} \tilde{\lambda}\right) \times r\right\|}
$$

with $\left(J_{\Omega}^{e} \tilde{\lambda}\right) \times r \neq 0$.

Let $\lambda_{V} \in \operatorname{Ker} J_{V}^{e}$. This implies that the left-hand side of (51) is $J_{V}^{e} \lambda_{V}=0$, so ensuring $\left(J_{\Omega}^{e} \lambda_{V}\right) \times r \neq 0$ guarantees that (51) does not hold. Since the joint axes of the manipulator do not intersect at the same point, $\operatorname{dimKer} J_{V} \leq 2$ by Lemma 1. However, because of Lemma $2, J_{\Omega}^{e} \lambda_{V} \neq 0$ for any nonzero $\lambda_{V} \in \operatorname{Ker} J_{V}^{e}$. Since $\operatorname{Ker} J_{V}^{e}$ is a subspace, and its dimension is not greater than two, the set $\left\{J_{\Omega}^{e} \lambda_{V}: \lambda_{V} \in \operatorname{Ker} J_{V}^{e}\right\}$ is a plane, a line or a point containing the origin (in the last case it is the origin itself, however it only happens in the trivial $\lambda_{V}=0$ case). If there exists $\lambda_{V} \neq 0$, then $r$ needs to be chosen such that it has a nonzero component perpendicular to $\left\{J_{\Omega}^{e} \lambda_{V}: \lambda_{V} \in \operatorname{Ker} J_{V}^{e}\right\}$, that implies $\left(J_{\Omega}^{e} \lambda_{V}\right) \times r \neq 0$, and (54) yields $\gamma=0$ (by substituting $\left.\tilde{\lambda}:=\lambda_{V}\right)$. So if there exists $\lambda_{V} \neq 0$, and $r$ has a nonzero component perpendicular to $\left\{J_{\Omega}^{e} \lambda_{V}: \lambda_{V} \in \operatorname{Ker} J_{V}^{e}\right\}$, then choosing $\gamma \neq 0$ guarantees that (51) does not hold for any nontrivial $\lambda_{V} \in \operatorname{Ker} J_{V}^{e}$.

Let $S=\left(\operatorname{Ker} J_{V}^{e}\right)^{\perp}$ be the orthogonal complement of $\operatorname{Ker} J_{V}^{e}$, i.e. $S$ is a subspace of $\mathbb{R}^{3}, S \cup \operatorname{Ker} J_{V}^{e}=\mathbb{R}^{3}$ and every vector from $S$ is orthogonal to every vector from $\operatorname{Ker} J_{V}^{e}$. Thus any vector from $\mathbb{R}^{3}$ can be written as $\lambda=c_{V} \lambda_{V}+c_{S} \lambda_{S}$, with $\lambda_{V} \in \operatorname{Ker} J_{V}^{e}$, $\lambda_{S} \in S$ for some $c_{V}, c_{S} \in \mathbb{R}$.

Suppose that $\operatorname{dimKer} J_{V}^{e}=2$. This yields that $\operatorname{dim} \operatorname{Ran} J_{V}^{e}=1$ and $\operatorname{dim} S=1$, thus the set $\left\{J_{V}^{e} \lambda_{S}: \lambda_{S} \in S\right\}$ is a one-dimensional subspace (a line passing through the origin) in the image space of $J_{V}^{e}$. However $r$ also has a component in the image space of $J_{V}^{e}$ because of Theorem 2, and since the image space 
is one-dimensional, $\left\langle J_{V}^{e} \lambda_{S}, r\right\rangle \neq 0$ for any nonzero $\lambda_{S} \in S$ and nonzero $r \in \mathbb{R}^{3}$, thus (52) can not hold for any nonzero $\lambda_{S} \in S$. Since $J_{V}^{e}\left(c_{V} \lambda_{V}+c_{S} \lambda_{S}\right)=c_{S} J_{V}^{e} \lambda_{S}$, this concludes the proof if $\operatorname{dimKer} J_{V}^{e}=2$.

Now examine the case when $\operatorname{dimKer} J_{V}^{e}<2$. Note that if $Q=0$, then the manipulator has the architecture and joint configuration as one of the three examples in Fig. 5, thus $\operatorname{rank} J_{V}^{e}=1$ yielding $\operatorname{dimKer} J_{V}^{e}=2$ if the end effector points are located the same way as in Fig. 5. If the manipulator has the same architecture and joint configuration as one of the examples in Fig. 5, but the end effector point is located such that the rank of the task Jacobian is greater than one, then the end effector point can be relocated to have $\operatorname{rank} J_{V}^{e}=1$, so we get the $\operatorname{dimKer} J_{V}^{e}=2$ again. The vector we used to relocate the end effector point then can be subtracted from the regularization vector to get the same manipulator architecture after the regularization. So without the loss of generality, we may assume that if $\operatorname{dimKer} J_{V}^{e}<2$, then $Q \neq 0$.

Notice that because of the definition of matrix transpose, (53) can be rearranged to get

$$
\left\langle\lambda,\left(J_{V}^{e}\right)^{\top} J_{\Omega}^{e} \lambda\right\rangle=0
$$

Since in a homogeneous quadratic form the matrix can be replaced with its symmetrical part, (55) can be written as

$$
\left\langle\lambda, 1 / 2\left(\left(J_{V}^{e}\right)^{\top} J_{\Omega}^{e}+J_{V}^{e}\left(J_{\Omega}^{e}\right)^{\top}\right) \lambda\right\rangle=0,
$$

and because of the definition of $Q$, this equation simplifies to the quadratic form

$$
\langle\lambda, Q \lambda\rangle=0
$$

Suppose that $\operatorname{dimKer} J_{V}^{e}=1$. Then the condition (52) becomes

$$
\left\langle J_{V}^{e}\left(c_{V} \lambda_{V}+c_{S} \lambda_{S}\right), r\right\rangle=\left\langle J_{V}^{e} c_{S} \lambda_{S}, r\right\rangle=0
$$

Since $\operatorname{dimKer} J_{V}^{e}=1 \mathrm{implies} \operatorname{dim} \operatorname{Ran} J_{V}^{e}=2$, and $\operatorname{dim} S=2$, the set $\left\{J_{V}^{e} \lambda_{S}: \lambda_{S} \in S\right\} \subseteq \operatorname{Ran} J_{V}^{e}$ is a two-dimensional subspace, while $r \in \operatorname{Ran} J_{V}^{e}$ is a vector in the image space of $J_{V}^{e}$, the set $\tilde{\lambda}_{S}$ for which (58) holds is a one-dimensional subspace, lying in $\operatorname{Ran} J_{V}^{e}$ being orthogonal to the vector $\left(J_{V}^{e}\right)^{\top} r$. Since $\tilde{\lambda}_{S}$ is a one-dimensional subspace, it is a line passing through the origin. Identify $\tilde{\lambda}_{S}$ with the direction vector of this line. Thus condition (57) needs to be examined only on the two-dimensional subspace spanned by the vectors $\tilde{\lambda}_{S}$ and $\lambda_{V}$. Let this subspace be denoted by $\tilde{S}$. The solution of the quadratic equation (57) restricted to $\tilde{S}$ by Lemma 4 is either two lines, one line, or a point, all containing the origin. If there is a nontrivial solution (two lines or one line), then let $\tilde{\lambda}$ be the set of the unit direction vectors of the lines. Then if $\gamma$ is chosen such that (54) does not hold for this $\tilde{\lambda}$, then (51) does not hold, and this concludes the proof if $\operatorname{dimKer} J_{V}^{e}=1$.
Suppose that $\operatorname{dimKer} J_{V}^{e}=0$. In this case $\operatorname{dim} S=3$, and $r$ can be chosen arbitrarily. Let $r \in \mathbb{R}^{3}$ be fixed. Then the condition (52) is only satisfied on the set $\left\{\tilde{\lambda}_{S}: \tilde{\lambda}_{S} \in S, \tilde{\lambda}_{S} \perp\left(J_{V}^{e}\right)^{\top} r\right\}$, that is a two-dimensional subspace of $\mathbb{R}^{3}$, denote it by $\tilde{S}$. Then the solution of (57) restricted to $\tilde{S}$ is either two lines, a line or a point containing the origin by Lemma 4 . If there is a nontrivial solution (two lines or one line), then let $\tilde{\lambda}$ be the set of the unit direction vectors of the lines. Then if $\gamma$ is chosen such that (54) does not hold for this $\tilde{\lambda}$, then (51) does not hold, and this concludes the proof if $\operatorname{dimKer} J_{V}^{e}=0$.

Note that due to Theorem 1 the ranks of $J^{e}, J^{s}$ and $J^{b}$ are the same in every joint configuration, so the Jacobian $J^{e}$ can be replaced with $J^{s}$ or $J^{b}$ in the conditions of Theorem 5 .

Given a regularization vector $r \in \mathbb{R}^{3}$ and a number $\gamma \in \mathbb{R}$ , the question arises that how efficient the regularization is. In the following Theorem, we give bounds on the singular values of the regularized Jacobian.

Theorem 6. Let $L$ be the length of the 3R robot arm in a fully extended state. The $\bar{\sigma}$ largest singular value of the regularized Jacobian can be upper bounded and the $\underline{\sigma}$ smallest singular value of the regularized Jacobian can be lower bounded as

$$
\begin{gathered}
\bar{\sigma}\left(J^{\mathrm{reg}}\right) \leq 3 \sqrt{L^{2}+\gamma^{2}} \\
\underline{\sigma}\left(J^{\mathrm{reg}}\right) \geq \frac{\left|\operatorname{det} J^{\mathrm{reg}}\right|}{9\left(L^{2}+\gamma^{2}\right)}
\end{gathered}
$$

provided that the regularization vector is a unit vector.

Proof. Since the columns of the regularized Jacobian have the form $v_{i}+\gamma \omega_{i} \times r$, with $\omega_{i}$ being a unit vector, and $r$ is also a unit vector, the length of $v_{i}$ is not greater than $L$, while the length of $\gamma \omega_{i} \times r$ is not greater than $\gamma$, the length of the vector $v_{i}+\gamma \omega_{i} \times r$ is not greater than $\sqrt{L^{2}+\gamma^{2}}$. This implies that the absolute value of the coordinates of the columns of the regularized Jacobian can not exceed $\sqrt{L^{2}+\gamma^{2}}$, thus both the maximal absolute value column sum and maximal absolute value row sum of the matrix are not greater than $3\left(\sqrt{L^{2}+\gamma^{2}}\right)$, thus

$$
\begin{gathered}
\left\|J^{\text {reg }}\right\|_{1} \leq 3\left(\sqrt{L^{2}+\gamma^{2}}\right) \\
\left\|J^{\text {reg }}\right\|_{\infty} \leq 3\left(\sqrt{L^{2}+\gamma^{2}}\right) .
\end{gathered}
$$

Since $\bar{\sigma}\left(J^{\text {reg }}\right) \leq\left(\left\|J^{\text {reg }}\right\|_{1} \| J^{\text {reg } \|_{\infty}}\right)^{1 / 2}$ that can be found e.g. in the work of Turkmen and Civciv [23],

$$
\bar{\sigma}\left(J^{\text {reg }}\right) \leq 3 \sqrt{L^{2}+\gamma^{2}} .
$$

Let the singular values of the matrix be $\bar{\sigma}\left(J^{\text {reg }}\right) \geq$ $\tilde{\sigma}\left(J^{\text {reg }}\right) \geq \underline{\sigma}\left(J^{\text {reg }}\right)$, with $\tilde{\sigma}\left(J^{\text {reg }}\right)$ being the middle singular value. Since the determinant of the matrix is $\left|\operatorname{det} J^{\text {reg }}\right|=$ $\bar{\sigma}\left(J^{\text {reg }}\right) \tilde{\sigma}\left(J^{\text {reg }}\right) \underline{\sigma}\left(J^{\text {reg }}\right)$, and

$$
\bar{\sigma}\left(J^{\mathrm{reg}}\right) \tilde{\sigma}\left(J^{\mathrm{reg}}\right) \underline{\sigma}\left(J^{\mathrm{reg}}\right) \leq \bar{\sigma}^{2}\left(J^{\mathrm{reg}}\right) \underline{\sigma}\left(J^{\mathrm{reg}}\right),
$$


it follows for $\underline{\sigma}\left(J^{\text {reg }}\right)$ that the inequality

$$
\underline{\sigma}\left(J^{\mathrm{reg}}\right) \geq \frac{\left|\operatorname{det} J^{\mathrm{reg}}\right|}{\bar{\sigma}^{2}\left(J^{\mathrm{reg}}\right)}
$$

holds, and substituting (63) results in

$$
\underline{\sigma}\left(J^{\mathrm{reg}}\right) \geq \frac{\left|\operatorname{det} J^{\mathrm{reg}}\right|}{9\left(L^{2}+\gamma^{2}\right)} .
$$

According to Theorem 6, the smallest singular value of the regularized Jacobian is lower bounded by the absolute value of its determinant divided by a term quadratic in $\gamma$ as shown in (60). We will show in the following theorem that the determinant of the regularized Jacobian is at most quadratic in $\gamma$.

Theorem 7. The determinant of the regularized Jacobian of a $3 \mathrm{R}$ robot can be expressed as

$$
\begin{aligned}
\operatorname{det} J^{\mathrm{reg}} & =v_{1}^{e} \cdot\left(v_{2}^{e} \times v_{3}^{e}\right) \\
& +\gamma\left(r \times v_{1}^{e}\right) \cdot\left(\xi_{2}^{e} \times_{6} \xi_{3}^{e}\right)_{V} \\
& -\gamma\left(r \times v_{2}^{e}\right) \cdot\left(\xi_{1}^{e} \times{ }_{6} \xi_{3}^{e}\right)_{V} \\
& +\gamma\left(r \times v_{3}^{e}\right) \cdot\left(\xi_{1}^{e} \times{ }_{6} \xi_{2}^{e}\right)_{V} \\
& +\gamma^{2}\left(r \cdot v_{1}^{e}\right)\left(r \cdot\left(\xi_{2}^{e} \times_{6} \xi_{3}^{e}\right)_{\Omega}\right) \\
& -\gamma^{2}\left(r \cdot v_{2}^{e}\right)\left(r \cdot\left(\xi_{1}^{e} \times_{6} \xi_{3}^{e}\right)_{\Omega}\right) \\
& +\gamma^{2}\left(r \cdot v_{3}^{e}\right)\left(r \cdot\left(\xi_{1}^{e} \times{ }_{6} \xi_{2}^{e}\right)_{\Omega}\right) .
\end{aligned}
$$

Proof.

The columns of the regularized Jacobian are

$$
v_{i}^{\mathrm{reg}}=v_{i}^{e}+\gamma \omega_{i}^{e} \times r, \quad i=1,2,3 .
$$

Using the following property of the determinant, i.e.

$$
\operatorname{det}(a, b+d, c)=\operatorname{det}(a, b, c)+\operatorname{det}(a, d, c),
$$

the determinant of $J^{\mathrm{reg}}$ can be written as

$$
\begin{aligned}
\operatorname{det} J^{\mathrm{reg}} & =\operatorname{det}\left(v_{1}^{e}, v_{2}^{e}, v_{3}^{e}\right) \\
& +\gamma \operatorname{det}\left(v_{1}^{e}, v_{2}^{e}, \omega_{3}^{e} \times r\right) \\
& +\gamma \operatorname{det}\left(v_{1}^{e}, \omega_{2}^{e} \times r, v_{3}^{e}\right) \\
& +\gamma \operatorname{det}\left(\omega_{1}^{e} \times r, v_{2}^{e}, v_{3}^{e}\right) \\
& +\gamma^{2} \operatorname{det}\left(\omega_{1}^{e} \times r, \omega_{2}^{e} \times r, v_{3}^{e}\right) \\
& +\gamma^{2} \operatorname{det}\left(\omega_{1}^{e} \times r, v_{2}^{e}, \omega_{3}^{e} \times r\right) \\
& +\gamma^{2} \operatorname{det}\left(v_{1}^{e}, \omega_{2}^{e} \times r, \omega_{3}^{e} \times r\right) .
\end{aligned}
$$

Using another property of the determinant of $3 \times 3$ matrices, i.e.

$$
\operatorname{det}(a, b, c)=a \cdot(b \times c)=c \cdot(a \times b)=-b \cdot(a \times c),
$$

the expression for $\operatorname{det} J^{\mathrm{reg}}$ can be further manipulated to get

$$
\begin{aligned}
\operatorname{det} J^{\mathrm{reg}} & =v_{1}^{e} \cdot\left(v_{2}^{e} \times v_{3}^{e}\right) \\
& -\gamma v_{2}^{e} \cdot\left(v_{1}^{e} \times\left(\omega_{3}^{e} \times r\right)\right) \\
& +\gamma v_{3}^{e} \cdot\left(v_{1}^{e} \times\left(\omega_{2}^{e} \times r\right)\right) \\
& +\gamma v_{2}^{e} \cdot\left(v_{3}^{e} \times\left(\omega_{1}^{e} \times r\right)\right) \\
& +\gamma^{2} v_{3}^{e} \cdot\left(\left(\omega_{1}^{e} \times r\right) \times\left(\omega_{2}^{e} \times r\right)\right) \\
& -\gamma^{2} v_{2}^{e} \cdot\left(\left(\omega_{1}^{e} \times r\right) \times\left(\omega_{3}^{e} \times r\right)\right) \\
& +\gamma^{2} v_{1}^{e} \cdot\left(\left(\omega_{2}^{e} \times r\right) \times\left(\omega_{3}^{e} \times r\right)\right) .
\end{aligned}
$$

The result is acquired with the application of some identities from vector algebra, and by introducing the notation $\left(\xi_{i} \times{ }_{6} \xi_{j}\right)_{V}=: \omega_{i} \times v_{j}-\omega_{j} \times v_{i}$ and $\left(\xi_{i} \times \xi_{6}\right)_{\Omega}=: \omega_{i} \times \omega_{j}$

Using the expressions (60) and (67) in Theorems 6 and 7, one may search the $\gamma \in \mathbb{R}$ and $r \in \mathbb{R}^{3}$ pairs that maximize the smallest singular value of the regularized Jacobian. However, we will not discuss this maximization problem in this paper.

\section{Application of the regularization for stable CLIK algorithm}

In this section we discuss the application of the regularization method in the closed-loop inverse kinematics (CLIK) algorithm

$$
\theta[k+1]=\theta[k]+T J^{\#}(\theta[k])\left(\dot{d}[k]+\alpha\left(d[k]-f_{p}(\theta[k])\right)\right)
$$

where $\theta[k]$ is the joint variable vector in the $k$ th step, $T$ is the sampling time, $J^{\sharp}(\theta[k])$ is the pseudoinverse of the task Jacobian in the joint configuration $\theta[k], \dot{d}[k]$ is the desired end effector velocity in the $k$ th step, $d[k]$ is the desired end effector position in the $k$ th step, while $f_{p}(\theta[k])$ is the real end effector position in the $k$ th step and $\alpha$ is a gain parameter of the algorithm.

In the work of Falco and Natale [20] it was shown that this algorithm is asymptotically stable if the following three assumptions are true

1. $\exists \delta>0: \forall \theta \in \mathbb{R}^{n},\|J(\theta)\| \leq \delta$;

2. $\exists \beta>0: \forall \theta \in \mathbb{R}^{n}, \underline{\sigma}\left(J(\theta) J^{\top}(\theta)\right) \geq \beta$;

3. For the remainder term $r(\theta, \tilde{\theta})$ in the first-order Taylor-series approximation of the positioning problem

$$
f_{p}(\theta+\tilde{\theta})=f_{p}(\theta)+J(\theta) \tilde{\theta}+r(\theta, \tilde{\theta})
$$

it is true, that $\exists v>0: \forall \theta \in R^{n},\|r(\theta, \tilde{\theta})\| \leq v\|\tilde{\theta}\|^{2}$; and the conditions

$$
0<\alpha<1 / T
$$

$$
\left\|e_{0}\right\|<\frac{1}{\alpha T v \delta^{\prime 2}}
$$


are satisfied with $\delta^{\prime}=\delta / \beta$. Note that the norms used in the assumptions and conditions are 2-norms.

We will show that with the application of the regularization, it can be guaranteed that the assumptions are true if the task Jacobian of the manipulator is regularizable. By Theorem 6, the largest singular value of the regularized Jacobian can be upper bounded, implying that

$$
\delta=9\left(L^{2}+\gamma^{2}\right)
$$

is a sufficient upper bound for the largest singular value of $J^{\text {reg }}$ (i.e. the 2-norm of the matrix $J^{\text {reg }}$ ) that is a positive finite number if $\gamma$ is finite, so the first assumption is satisfied. Using the lower bound for the smallest singular value of the regularized Jacobian, it can be shown, that

$$
\beta=\left(\frac{\left|\operatorname{det} J^{\mathrm{reg}}\right|}{9\left(L^{2}+\gamma^{2}\right)}\right)^{2}
$$

is a sufficient lower bound for the smallest singular value of $J^{\mathrm{reg}}\left(J^{\mathrm{reg}}\right)^{\top}$ that is a nonzero positive number, since $\operatorname{det} J^{\mathrm{reg}}$ is not zero if the task Jacobian can be regularized and $r \in \mathbb{R}^{3}$ and $\gamma \in \mathbb{R}$ are chosen appropriately.

The second order approximation [5] of the positioning problem is

$$
\begin{aligned}
f_{p}(\theta[k]) & =\sum_{n=1}^{3} \xi_{n} \dot{\theta}_{n}[k]+ \\
& \frac{1}{2 !} \sum_{1 \leq l<m \leq 3} \dot{\theta}_{l}[k]\left(\xi_{l} \times_{6} \xi_{m}\right)_{V} \dot{\theta}_{m}[k]+\ldots
\end{aligned}
$$

In the paper of Falco and Natale [20] it was shown that if $v_{i}$ is the upper bound for the 2-norm of the Hessian of the $i$ th component of a function $f_{p}$, then $v \leq \sqrt{3} / 2 \max _{i} v_{i}$, with $v$ being the 2-norm of the Hessian of the function $f_{p}$. The Hessian for the $x, y, z$ components of the function $f_{p}$ are the matrices

$$
\begin{aligned}
& H_{x}=1 / 2\left[\left(\xi_{i} \times_{6} \xi_{j}\right)_{x}\right]_{i, j} \\
& H_{y}=1 / 2\left[\left(\xi_{i} \times_{6} \xi_{j}\right)_{y}\right]_{i, j} \\
& H_{z}=1 / 2\left[\left(\xi_{i} \times{ }_{6} \xi_{j}\right)_{z}\right]_{i, j}
\end{aligned}
$$

with $i, j=1,2,3$, where $\left(\xi_{i} \times{ }_{6} \xi_{j}\right)_{x}$ is the $x$ linear velocity component of the Lie-bracket of the generators $\xi_{i}$ and $\xi_{j},\left(\xi_{i} \times{ }_{6} \xi_{j}\right)_{y}$ is the $y$ linear velocity component of the Lie-bracket of the generators $\xi_{i}$ and $\xi_{j}$ and $\left(\xi_{i} \times{ }_{6} \xi_{j}\right)$ is the $z$ linear velocity component of the Lie-bracket of the generators $\xi_{i}$ and $\xi_{j}$. Let the length of the fully extended manipulator be $L$, then the absolute value of any component of the linear velocity generators of the Lie-brackets can not be greater than $2 L$, so for the 1-norm and $\infty$-norm of each Hessians we have that they are upper bounded by $2 \cdot 1 / 2 \cdot 2 L=2 L$, since the Hessians have zero diagonal, so there are at most two nonzero elements in each row and column, so the norm of each Hessian can not be greater than $2 L$. Thus the norm for the Hessian is upper bounded by

$$
v \leq \sqrt{3} L
$$

that is a finite number, and independent of the regularization, so the third assumption is also satisfied. The condition number of $J^{\mathrm{reg}}\left(J^{\mathrm{reg}}\right)^{\top}$ is upper bounded by

$$
\delta^{\prime}=\frac{\delta}{\beta}=\frac{\left(9\left(L^{2}+\gamma^{2}\right)\right)^{3}}{\left(\operatorname{det} J^{\mathrm{reg}}\right)^{2}}
$$

and thus

$$
\delta^{\prime} v \leq \sqrt{3} L \frac{\left(9\left(L^{2}+\gamma^{2}\right)\right)^{3}}{\left(\operatorname{det} J^{\mathrm{reg}}\right)^{2}}
$$

Since these numbers are all finite if $\operatorname{det} J^{\text {reg }} \neq 0$, the gain parameter $\alpha$ can be chosen such that the CLIK algorithm (73) is stable.

\section{Application of the regularization on an elbow manipulator}

Consider an elbow manipulator in the singular home configuration in Fig. 6. The geometric parameters of the manipulator are

$$
\begin{aligned}
& \omega_{1}=\left(\begin{array}{l}
0 \\
0 \\
1
\end{array}\right) \quad \omega_{2}=\left(\begin{array}{l}
1 \\
0 \\
0
\end{array}\right) \quad \omega_{3}=\left(\begin{array}{l}
1 \\
0 \\
0
\end{array}\right) \\
& q_{1}=\left(\begin{array}{l}
0 \\
0 \\
0
\end{array}\right) \quad q_{2}=\left(\begin{array}{l}
0 \\
0 \\
0
\end{array}\right) \quad q_{3}=\left(\begin{array}{l}
0 \\
0 \\
l_{1}
\end{array}\right)
\end{aligned}
$$

and the end effector position in the home configuration is

$$
p(0)=\left(\begin{array}{c}
0 \\
0 \\
l_{1}+l_{2}
\end{array}\right) \text {. }
$$

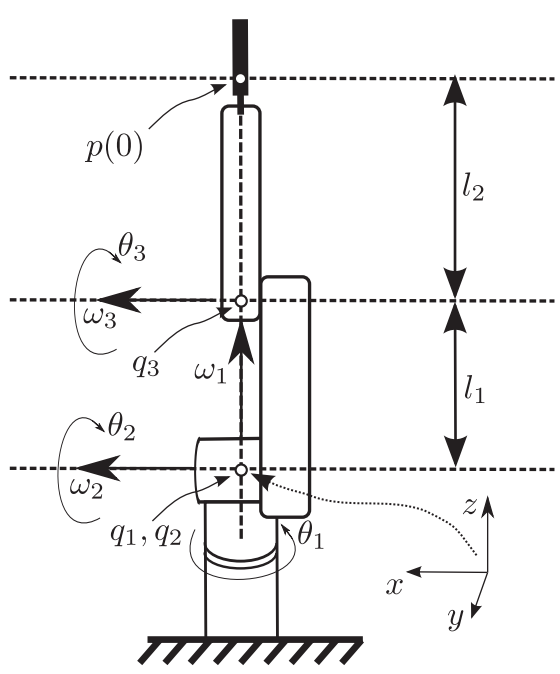

Fig. 6 Elbow manipulator in the singular home configuration, the origin of the base frame is in the point $q_{1}$. 
The end effector Jacobian in the home configuration is

$$
J^{e}(0)=\left(\begin{array}{ccc}
0 & 0 & 0 \\
0 & -l_{1}-l_{2} & -l_{2} \\
0 & 0 & 0 \\
0 & 1 & 1 \\
0 & 0 & 0 \\
1 & 0 & 0
\end{array}\right) .
$$

The task is to move in the $-z$ direction to the point $d_{1}=$ $\left(0,0, l_{1}+l_{2} / 2\right)^{\top}$ (similar to the motion in Fig. 1), then move in the $x$ direction to the point $d_{2}=\left(l_{1} / 2,0, l_{1}+l_{2} / 2\right)^{\top}$ ( similar to the motion in Fig. 2), then move back to the initial singular configuration to the point $d_{3}=p(0)$.

The CLIK algorithm is run for 100 iterations, in the first 25 iterations the desired end effector position is $d_{1}$, in the next 25 iterations, the desired end effector position is $d_{2}$, and in the last 50 iterations the desired end effector position is $d_{3}$, i.e.

$$
d[k]= \begin{cases}d_{1} & \text { if } k \in[0,25) \\ d_{2} & \text { if } k \in[25,50) \\ d_{3} & \text { if } k \in[50,100]\end{cases}
$$

and $\dot{d}[k]=0$ for all $k$. The sampling time is considered to be unit, i.e. $T=1$. The link lengths are considered to be unit as well, i.e. $l_{1}=l_{2}=1$. The CLIK algorithm is carried out using the regularization method with the regularization vector being the normalized linear velocity generator of the third joint (recalculated in every joint configuration), i.e.

$$
r[k]=\frac{v_{3}^{e}(\theta[k])}{\left\|v_{3}^{e}(\theta[k])\right\|},
$$

and the parameter $\gamma$ is calculated as

$$
\gamma[k]=k_{1} \exp \left(-k_{2}|\operatorname{det} J(\theta[k])|\right)
$$

with $\operatorname{det} J(\theta[k])$ being the determinant of the task Jacobian (before the regularization) in the joint configuration $\theta[k]$. The parameters $k_{1}, k_{2}$ and the gain parameter $\alpha$ are chosen as $k_{1}=0.5, k_{2}=0.2, \alpha=0.9$. The CLIK is carried out according to (73), but $J$ is replaced with $J^{\text {reg }}$ defined by (19) in every joint configuration $\theta[k]$.

Theorem 8. Consider an elbow manipulator with the parameters given in (86)-(88). The inverse positioning problem of the elbow manipulator can be regularized in almost every joint configuration, if the regularization vector is chosen as the linear velocity generator corresponding to the last joint of the manipulator.

Proof. By Theorem 7, the determinant of the regularized task Jacobian can be written as

$$
\operatorname{det} J^{\mathrm{reg}}=v_{1} \cdot\left(v_{2} \times v_{3}\right)+\gamma \alpha+\gamma^{2} \beta,
$$

$$
\begin{gathered}
\alpha=\left(r \times v_{1}\right) \cdot\left(\omega_{2} \times v_{3}-\omega_{3} \times v_{2}\right) \\
-\left(r \times v_{2}\right) \cdot\left(\omega_{1} \times v_{3}-\omega_{3} \times v_{1}\right) \\
+\left(r \times v_{3}\right) \cdot\left(\omega_{1} \times v_{2}-\omega_{2} \times v_{1}\right), \\
\beta=\left(r \cdot v_{1}\right)\left(r \cdot\left(\omega_{2} \times \omega_{3}\right)\right) \\
-\left(r \cdot v_{2}\right)\left(r \cdot\left(\omega_{1} \times \omega_{3}\right)\right) \\
+\left(r \cdot v_{3}\right)\left(r \cdot\left(\omega_{1} \times \omega_{2}\right)\right) .
\end{gathered}
$$

Since the second and third joint axes are parallel in every joint configuration, this yields that $\omega_{2} \times \omega_{3}=0$ and $\omega_{2}=\omega_{3}$ in every configuration, thus $\beta$ simplifies to

$$
\begin{aligned}
\beta & =-\left(r \cdot v_{2}\right)\left(r \cdot\left(\omega_{1} \times \omega_{3}\right)\right)+\left(r \cdot v_{3}\right)\left(r \cdot\left(\omega_{1} \times \omega_{2}\right)\right) \\
& =r \cdot\left(v_{3}-v_{2}\right)\left(r \cdot\left(\omega_{1} \times \omega_{2}\right)\right) .
\end{aligned}
$$

Since $\xi_{2} \neq \xi_{3}$ holds in every joint configuration, it yields that $v_{3} \neq v_{2}$, thus $v_{3}-v_{2}$ is never zero. It is also true due to Theorem 4 , that $v_{3} \neq 0$, and since $r=v_{3} /\left\|v_{3}\right\|, r \cdot\left(v_{3}-v_{2}\right)$ is never zero. So $\beta$ is zero if and only if $r \cdot\left(\omega_{1} \times \omega_{2}\right)=0$. This expression can be reformulated as

$$
r \cdot\left(\omega_{1} \times \omega_{2}\right)=-\omega_{1} \cdot\left(r \times \omega_{2}\right)=\omega_{2} \cdot\left(r \times \omega_{1}\right) .
$$

Since $v_{3}=\omega_{3} \times\left(p-q_{3}\right)$, and $\omega_{2}=\omega_{3}, v_{3}$ is orthogonal to $\omega_{2}$, thus $r \perp \omega_{2}$, and $r \times \omega_{2} \neq 0$. Since $\omega_{2} \perp \omega_{1}$ as well, $\omega_{2} \cdot\left(r \times \omega_{1}\right) \neq 0$, if $r \times \omega_{1} \neq 0$. So $\beta=0$ if and only if $r \times \omega_{1}=0$, so if $r$ is parallel to $\omega_{1}$. This only happens if $\theta_{2}= \pm \pi / 2$ and $\theta_{3}=0$ or $\theta_{3}= \pm \pi$. Note that in the first case $\left(\theta_{3}=0\right)$, the manipulator is fully extended, while in the latter case $\left(\theta_{3}= \pm \pi\right)$, the manipulator is folded back.

In these configurations $\beta=0$, however $\alpha \neq 0$ may hold. So examine the value of $\alpha$, when $r \times \omega_{1}=0$, so we can suppose that $r=\omega_{1}$. The value of $\alpha$ is

$$
\begin{gathered}
\alpha=\left(\omega_{1} \times v_{1}\right) \cdot\left(\omega_{2} \times v_{3}-\omega_{3} \times v_{2}\right) \\
-\left(\omega_{1} \times v_{2}\right) \cdot\left(\omega_{1} \times v_{3}-\omega_{3} \times v_{1}\right) \\
+\left(\omega_{1} \times v_{3}\right) \cdot\left(\omega_{1} \times v_{2}-\omega_{2} \times v_{1}\right) .
\end{gathered}
$$

Utilizing that $\omega_{2}=\omega_{3}$. and $r=v_{3} /\left\|v_{3}\right\|=\omega_{1}$, thus $\omega_{1} \times v_{3}=0$, the expression for $\alpha$ simplifies to

$$
\begin{aligned}
& \alpha=\left(\omega_{1} \times v_{1}\right) \cdot\left(\omega_{2} \times\left(v_{3}-v_{2}\right)\right) \\
& -\left(\omega_{1} \times v_{2}\right) \cdot\left(\omega_{1} \times v_{3}-\omega_{3} \times v_{1}\right) \\
& =\left(\omega_{1} \times v_{1}\right) \cdot\left(\omega_{2} \times v_{3}\right) \\
& -\left(\omega_{1} \times v_{1}\right) \cdot\left(\omega_{2} \times v_{2}\right) \\
& +\left(\omega_{1} \times v_{2}\right) \cdot\left(\omega_{3} \times v_{1}\right) .
\end{aligned}
$$

Using the identity $(a \times b) \cdot(c \times d)=b^{\top}\left(\left(c^{\top} a\right) I-c a^{\top}\right) d$, we get

with 


$$
\begin{gathered}
\alpha=v_{1}^{\top}\left(\omega_{2}^{\top} \omega_{1} I-\omega_{2} \omega_{1}^{\top}\right) v_{3} \\
-v_{1}^{\top}\left(\omega_{2}^{\top} \omega_{1} I-\omega_{2} \omega_{1}^{\top}\right) v_{2} \\
-v_{2}^{\top}\left(\omega_{3}^{\top} \omega_{1} I-\omega_{3} \omega_{1}^{\top}\right) v_{1} \\
=v_{1}^{\top} \omega_{2} \omega_{1}^{\top}\left(v_{3}-v_{2}\right),
\end{gathered}
$$

where we have used the facts that $\omega_{2}^{\top} \omega_{1}=\omega_{3}^{\top} \omega_{1}=0$, and $\omega_{1}^{\top} v_{1}=0$. We have already seen that $v_{3}-v_{2} \neq 0$, and since in the analyzed configuration $v_{3}$ is parallel to $\omega_{1}, \omega_{1}^{\top}\left(v_{3}-v_{2}\right) \neq 0$. So the question is whether $v_{1}^{\top} \omega_{2}$ is zero or not. Thus in the analyzed configuration, $\alpha=0$ if and only if $v_{1}^{\top} \omega_{2}=0$. Due to its definition, $v_{1}$ may be written as $\omega_{1} \times\left(p-q_{1}\right)$, where $p$ isthe end effector point in the current configuration, and $q_{1}$ is a point on the first joint axis. So we need to further analyze the expression

$$
\left(\omega_{1} \times\left(p-q_{1}\right)\right) \cdot \omega_{2}=\left(\omega_{2} \times\left(p-q_{2}\right)\right) \cdot \omega_{1}=v_{2} \cdot \omega_{1},
$$

where we have used that $q_{1}=q_{2}$. Since in the analyzed configuration, $v_{2}$ is parallel to $v_{3}$, and $v_{3}$ is parallel to $\omega_{1}, \omega_{1}$ is parallel to $v_{2}$, so $v_{2} \cdot \omega_{1}=0$ if and only if $v_{2}=0$, that holds if and only if $p=q_{2}$. This can happen only in the joint configurations $\theta_{2}= \pm \pi / 2, \theta_{3}= \pm \pi$, and only for manipulators whose links have equal length, i.e. $l_{1}=l_{2}$. In every other case either $\beta$ or $\alpha$ is not zero with $r=v_{3} /\left\|v_{3}\right\|$, so there exists a $\gamma \in R$ such that $\operatorname{det} J^{\text {reg }} \neq 0$ that concludes the proof.

The CLIK algorithm is also carried out using the DLS method and the modified LM method. When the DLS method is used, the pseudoinverse of the Jacobian is calculated as

$$
J^{\#}=J^{\top}\left(J J^{\top}+\lambda I\right)
$$

with $\lambda=0.1$ and $I$ being the $3 \times 3$ identity matrix. For the gain parameter the value $\alpha=0.5$ is used.

The update law for the joint variables using the modified LM method [16] is

$$
\theta[k+1]=\theta[k]+H_{k}^{-1} g_{k}
$$

with

$$
\begin{gathered}
H_{k}=J^{\top} W_{e} J+W_{n} \\
g_{k}=J^{\top} W_{e}\left(d[k]-f_{p}(\theta[k])\right)
\end{gathered}
$$

and the matrices $W_{e}$ and $W_{n}$ are chosen as $W_{e}=I$ and $W_{n}=\operatorname{diag}\left(d[k]-f_{p}(\theta[k])\right)+\bar{w}_{n} I$ with $\bar{w}_{n}=0.001$ following the recommendations from the paper of Sugihara [16].

In the first case, the simulation is initiated at the $\theta=(0,0,0)^{\top}$ home configuration. The resulting joint paths, joint velocities and path tracking errors of the CLIK algorithm are in Figs. 7, 8 and 9 respectively. The dashed lines correspond to the results of the CLIK algorithm using the proposed regularization method, the dotted lines correspond to the results of the CLIK algorithm using the DLS method, and the dash-dot lines correspond to the results of the CLIK algorithm using modified LM method.
Since the initial position error is $e(0)=(0,0,-0.5)^{\top}$ (see Fig. 9), the error vector is in the singular direction, and only the CLIK algorithm using the regularization method can generate joint motion. The CLIK algorithms using the DLS and LM methods does not generate any joint motion during the simulation process (see Fig. 8).

Only the CLIK algorithm using the proposed regularization method is able to generate motion in singular direction. In the beginning of the simulation, motion in the singular $-z$ direction is needed. This is the singularity of the planar subassembly of the manipulator, so this situation is similar to the one in Fig. 1, where motion in singular direction requires infinite joint velocities. However, the algorithm is able to generate motion in singular direction with finite joint velocities, as it is shown in Fig. 8. At the 25th iteration step, the desired end effector position is changed such that the manipulator has to move parallel to its second and third joint axes, similar to the situation in Fig. 2. This type of singularity results in discontinuous joint path, however Fig. 7 shows that the algorithm generates joint motion with continuous joint paths. After the 50th iteration, the end effector of the manipulator is moved back to the $p(0)$ initial position. This movement is done by extending the planar subassembly of the manipulator (joint variables $\theta_{2}$ and $\theta_{3}$ are approaching zero), and the first joint is not moved back to the initial joint configuration. The tracking errors in Fig. 9 show that the algorithm is stable, and zero tracking error is reached after relatively small number of iterations if the regularization method is applied. Note that the tracking errors of the DLS and LM methods are the differences of the actual desired end effector position and the initial end effector position, since they generate no motion.

In order to compare the regularization method to the DLS and LM methods in a situation when they generate joint motion, the simulation is run in a second scenario where the initial configuration of the manipulator is the $\theta=(0.1,0.1,0.1)^{\top}$ nonsingular joint configuration. In this case all of the methods generate motion, since the initial tracking error does not coincide with a singular direction. The resulting joint paths, joint velocities and tracking errors are in Figs. 10, 11 and 12 respectively.

Fig. 12 shows that the path tracking error of all of the methods converge to zero, so they are stable, and solve the inverse kinematics problem, however Fig. 10 shows that the DLS method converges slowly to the $d_{3}$ singular end effector position. It can be observed from Figs. 12 and 11 that the regularization method has faster convergence than the other methods while the amplitudes of the joint velocities are in the same range. The regularization and DLS methods find the same joint configurations for the solution of the inverse kinematics problem, however the LM method over-rotates the first joint after the 25 th step, and finds the solution $\theta_{1}=3 \pi / 2$ instead of $\theta_{1}=\pi / 2$, resulting in much greater joint speeds. 

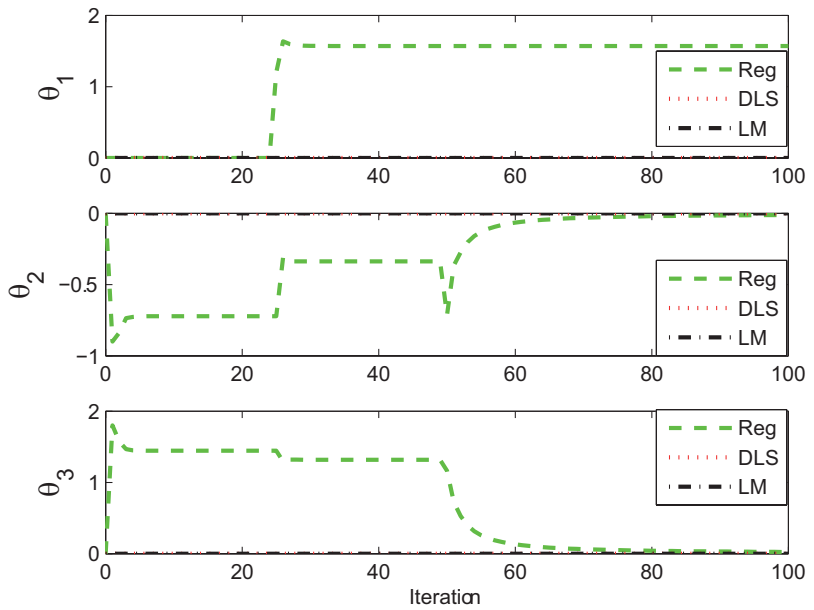

Fig. 7 The resulting joint path of the CLIK algorithm using the regularization (dashed lines), DLS (dotted lines) and modified LM methods (dash-dot lines), starting from the joint configuration $\theta=(0,0,0)^{\top}$.
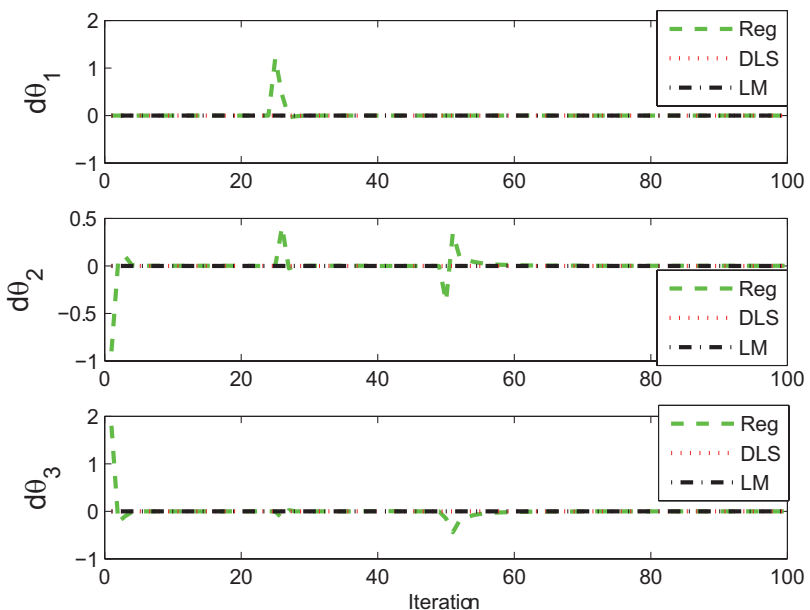

Fig. 8 The resulting joint velocities of the CLIK algorithm using the regularization (dashed lines), DLS (dotted lines) and modified LM methods

(dash-dot lines), starting from the joint configuration $\theta=(0,0,0)^{\top}$

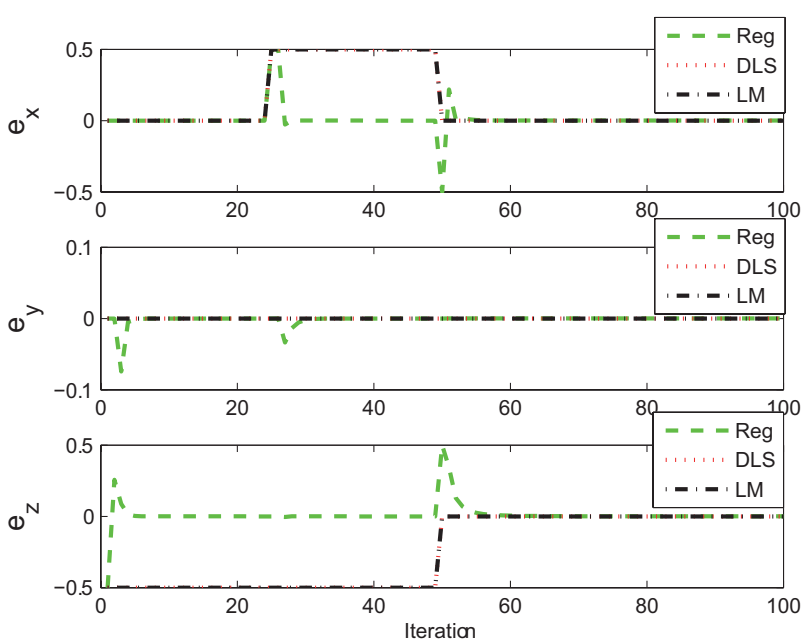

Fig. 9 The resulting path tracking errors of the CLIK algorithm using the regularization (dashed lines), DLS (dotted lines) and modified LM methods

(dash-dot lines), starting from the joint configuration $\theta=(0,0,0)^{\top}$.
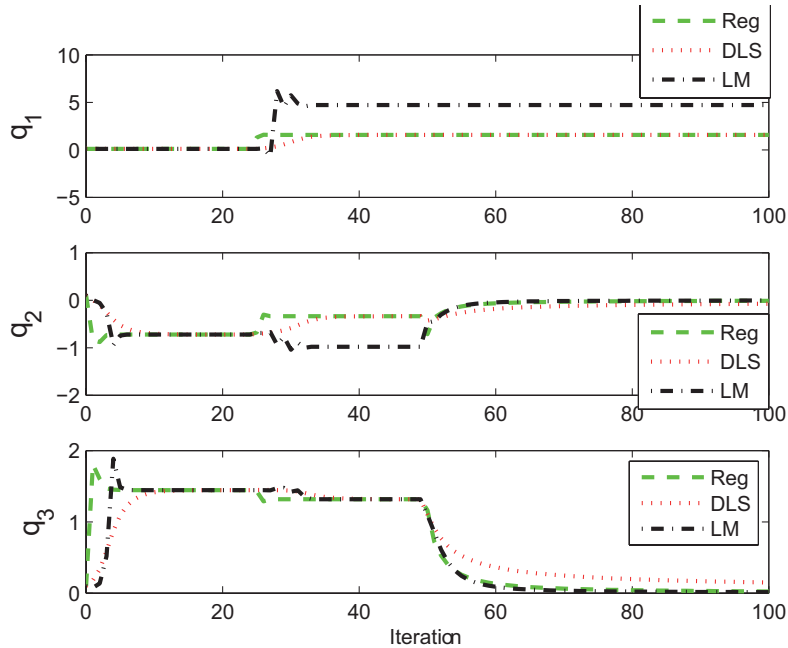

Fig. 10 The resulting joint path of the CLIK algorithm using the regularization (dashed lines), DLS (dotted lines) and modified LM methods (dash-dot lines), starting from the joint configuration $\theta=(0.1,0.1,0.1)^{\top}$.
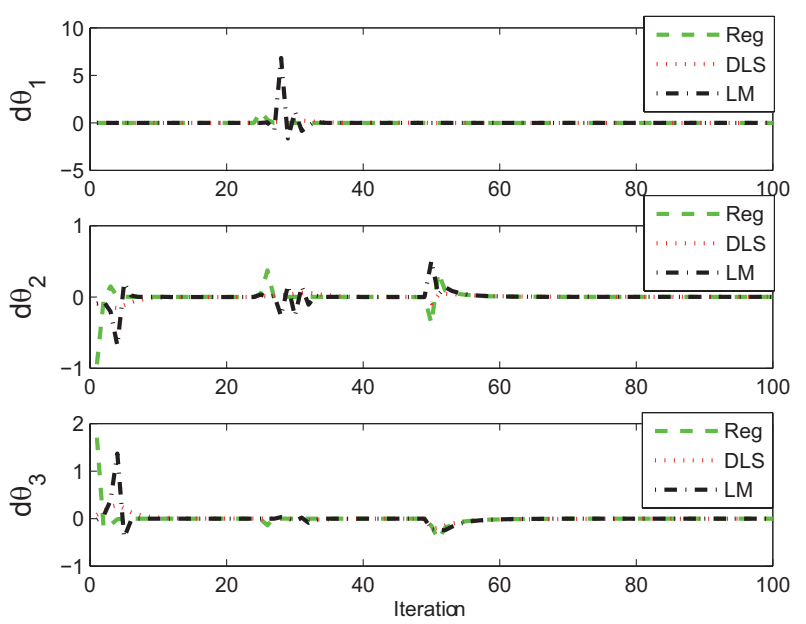

Fig. 11 The resulting joint velocities of the CLIK algorithm using the regularization (dashed lines), DLS (dotted lines) and modified LM methods (dash-dot lines), starting from the joint configuration $\theta=(0.1,0.1,0.1)^{\top}$.

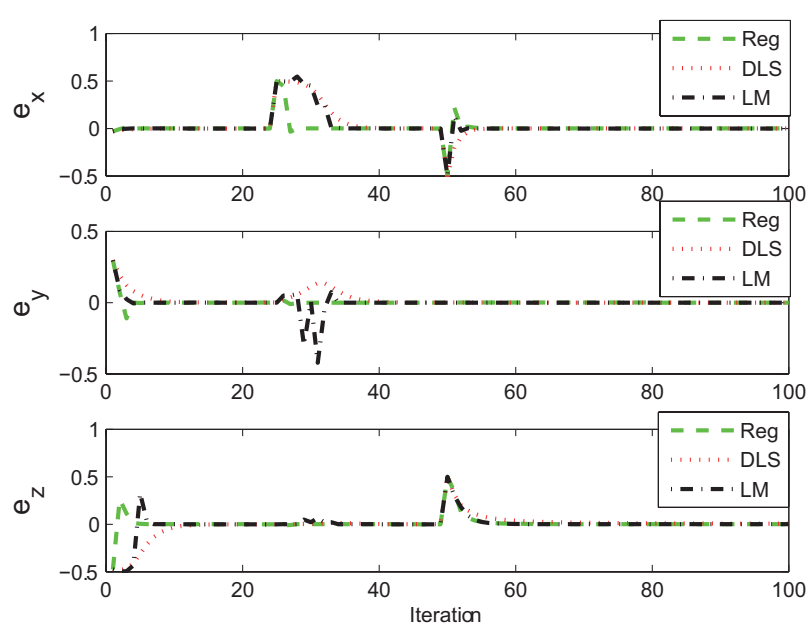

Fig. 12 The resulting path tracking errors of the CLIK algorithm using the regularization (dashed lines), DLS (dotted lines) and modified LM methods (dash-dot lines), starting from the joint configuration $\theta=(0.1,0.1,0.1)^{\top}$. 
In conclusion, if the initial joint configuration is nonsingular, all of the methods generate a joint path that is the solution of the inverse kinematics problem with similar properties, however if the initial joint configuration is singular, and the tracking error is in the singular direction, only the regularization method could generate joint motion.

\section{Conclusion}

The spatial inverse positioning problem of robot manipulators can be regularized, if the manipulator is capable of motion in the singular direction. After excluding the manipulator classes that can only move on surfaces, we can conclude that the spatial inverse positioning problem of a robot manipulator can be regularized, if its end effector Jacobian (or the spatial or body manipulator Jacobian) is full rank. This can be generalized easily for redundant robot manipulators, one only has to find a suitable $3 R$ subassembly of the manipulator whose end effector Jacobian is full rank.

The application of the regularization guarantees that the task Jacobian is full rank, thus the inverse mapping used in the CLIK algorithm is also full rank. The singular values of the task Jacobian depend on the regularization vector and the $\gamma$ parameter of the regularization. The appropriate choice of these parameters result in good numerical properties of the task Jacobian.

The formula for the determinant of the regularized Jacobian helps us find the appropriate choice of the regularization vector. This task becomes easy if the manipulator has special geometry, as it was the case with the elbow manipulator. Following the technique shown in this paper, the regularization vector may be constructed for other manipulators possessing special architecture as well.

The combination of the results with the stability theorem of the CLIK algorithm showed that with the application of the regularization technique, stable CLIK solutions can be acquired even if the manipulator has to face singular configurations. Simulation results showed that the regularization method generates motion in singular directions as well, opposed to the conventional numerical regularization techniques, like the DLS method or the LM method.

\section{References}

[1] Murray, R. M., Sastry, S. S., Li, Z. "A Mathematical Introduction to Robotic Manipulation." CRC Press, 1994.

[2] Selig, J. "Geometric Fundamentals of Robotics (Second Edition)." Springer, 2005.

[3] Siciliano, B., Sciavicco, L., Villani, L., Oriolo, G. "Robotics - Modelling, Planning and Control." Springer, 2009.

[4] Kim, Y.-J., Cheng, S., Kim, S., Iagnemma, K. "A stiffness-adjustable hyperredundant manipulator using a variable neutral-line mechanism for minimally invasive surgery." IEEE Transactions on Robotics. 30(2), pp. 382-395. 2014.

https://doi.org/10.1109/TRO.2013.2287975
[5] Donelan, P. S. "Singularity-theoretic methods in robot kinematics." Robotica. 25, pp. 641-659, 2007.

https://doi.org/10.1017/S0263574707003748

[6] Donelan, P., Müller, A. "Singularities of regional manipulators revisited." In: Advances in Robot Kinematics: Motion in Man and Machine (J. Lenarcic and M. M. Stanisic, eds.), pp. 509-519, Springer Netherlands, 2010. https://doi.org/10.1007/978-90-481-9262-5_55

[7] Kieffer, J. "Differential analysis of bifurcations and isolated singularities for robots and mechanisms." IEEE Transactions on Robotics and Automation. 10(1), pp. 1-10. 1994.

https://doi.org/10.1109/70.285580

[8] Watanabe, K., Sogen, K., Kawakami, N., Tachi, S. "An avoidance method of singular configurations in a master-slave system using intervening impedance." In: Proceedings of the 16th IEEE International Conference on Robot and Human Interactive Communication, pp. 1102-1107, 2007. https://doi.org/10.1109/ROMAN.2007.4415245

[9] McCarthy, J. M., Bodduluri, R. M. "Avoiding singular configurations in finite position synthesis of spherical 4r linkages." Mechanism and Machine Theory. 35, pp. 451-462, 2000. https://doi.org/10.1016/S0094-114X(99)00005-1

[10] Charles, I., Wampler, W. "Manipulator inverse kinematics solutions based on vector formulations and damped least-squares methods." IEEE Transactions on Systems, Man, and Cybernetics. SMC-16(1), pp. 93-101. 1986.

[11] Nakamura, Y., Hanafusa, H. "Inverse kinematic solutions with singularity robustness for robot manipulator control." Journal of Dynamic Systems, Measurement, and Control. 108(3), pp. 163-171. 1986. https://doi.org/10.1115/1.3143764

[12] Caccavale, F., Chiaverini, S., Siciliano, B. "Second-order kinematic control of robot manipulators with Jacobian damped least-squares inverse: Theory and experiments." IEEE/ASME Transactions on Mechatronics. 2(3), pp. 188-194, 1997.

[13] Chiaverini, S. "Singularity-robust task-priority redundancy resolution for real-time kinematic control of robot manipulators." IEEE Transactions on Robotics and Automation. 13(3), pp. 398-410. 1997. https://doi.org/10.1109/70.585902

[14] Tan, J., Xi, N., Wang, Y. "A singularity-free motion control algorithm for robot manipulators-a hybrid system approach." Automatica. 40(7), pp. 1239-1245, 2004.

https://doi.org/10.1016/j.automatica.2004.02.013

[15] Deo, A., Walker, I. D. "Adaptive nonlinear least squares for inverse kinematics." In: Proceesings of the IEEE International Conference on Robotics and Automation, pp. 186-193, 1993.

https://doi.org/10.1109/ROBOT.1993.291981

[16] Sugihara, T. "Solvability-unconcerned inverse kinematics by the Levenberg-Marquardt method." IEEE Transactions on Robotics. 27(5), pp. 984-991, 2011.

https://doi.org/10.1109/TRO.2011.2148230

[17] Drexler, D. A., Harmati, I. "Regularized Jacobian for the differential inverse positioning problem of serial revolute joint manipulators." In: Proceedings of the IEEE International Symposium on Intelligent Systems and Informatics, (Subotica, Serbia), pp. 227-232, Sept. 2013. https://doi.org/10.1109/SISY.2013.6662575

[18] Drexler, D. A., Harmati, I. "Regularization of the inverse positioning problem of revolute joint manipulators." In: Proceedings of the Workshop on the Advances of Information Technology: WAIT 2015, pp. 83-95, 2015.

[19] Drexler, D. A. "Regularization of the differential inverse positioning problem of an elbow manipulator." In: Proceedings of the IEEE 13th International Symposium on Intelligent Systems and Informatics: SISY2015, pp. 289-294, 2015. https://doi.org/10.1109/SISY.2015.7325397 
[20] Falco, P., Natale, C. "On the stability of closed-loop inverse kinematics algorithms for redundant robots." IEEE Transactions on Robotics. 27(4), pp. 780-784. 2011.

https://doi.org/10.1109/TRO.2011.2135210

[21] Humphreys, J. E. "Introduction to Lie Algebras and Representation Theory". Springer Verlag, New York, 1972.

[22] Drexler, D. A., Harmati, I. "Regularization of the differential inverse orientation problem of serial revolute joint manipulators." In: Proceedings of the IEEE International Symposium on Applied Computational Intelligence and Informatics, (Timisoara, Romania), pp. 225-230, May 2014. https://doi.org/10.1109/SACI.2014.6840065

[23] Turkmen, R., Civciv, H. "Some bounds for the singular values of matrices." Applied Mathematical Sciences. 1(49), pp. 2443-2449. 2007. 